\title{
Liver sinusoid on a chip
}

\author{
Yu Du*, Ning $\mathrm{Li}^{*, \dagger}$, Mian Long*,†,1 \\ *Center of Biomechanics and Bioengineering, Key Laboratory of Microgravity (National \\ Microgravity Laboratory), and Beijing Key Laboratory of Engineered Construction and \\ Mechanobiology, Institute of Mechanics, Chinese Academy of Sciences, Beijing, China \\ ${ }^{\dagger}$ School of Engineering Sciences, University of Chinese Academy of Sciences, Beijing, China \\ ${ }^{1}$ Corresponding author: e-mail address: mlong@imech.ac.cn
}

\section{CHAPTER OUTLINE}

1 Introduction of Cell-Cell Interactions in Liver Sinusoidal Microenvironments..............2

1.1 Architecture and Cell Composition of the Liver Sinusoids..........................2

1.2 Cellular Interactions in the Liver Sinusoids During Fibrosis and

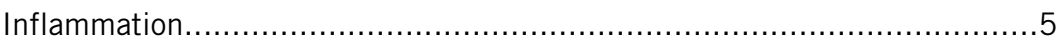

1.3 Sinusoidal Mechanical Microenvironments..........................................

1.4 In Vitro Models of the Liver Sinusoids................................................ 8

2 Construction of the Liver Sinusoid Chip..............................................................10

2.1 Microfluidic Device Fabrication................................................... 10

2.2 Primary Hepatic Cell Isolation....................................................... 11

2.3 Identification of Isolated Hepatic Cells............................................13

2.4 Chip Assembling and Characterization.............................................. 15

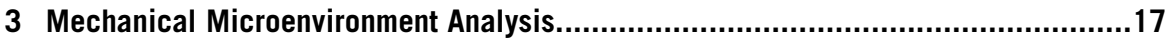

3.1 Fluidic Dynamic Model......................................................... 18

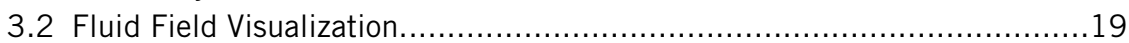

4 Liver-Specific Functions of the Sinusoidal Chip.................................................20

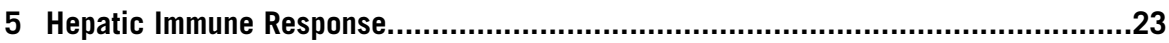

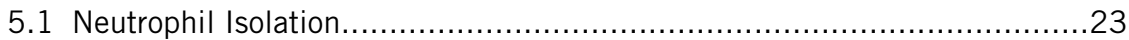

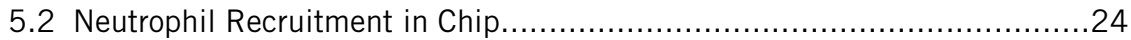

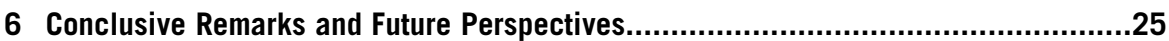

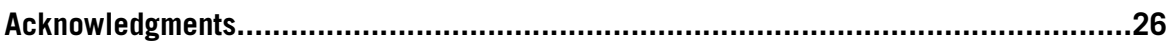

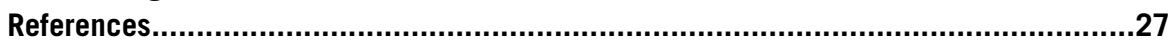

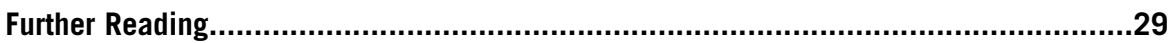




\section{Abstract}

Liver sinusoid is the main functional site in liver. Multiple types of hepatic cells are well organized in a precisely-controlled biochemical and biomechanical environment, maintaining a spectrum of hepatic functions. Here, using micro-engineering techniques, four types of primary hepatic cells are integrated into two layer channels connected by porous membrane, which recreates the sinusoidal cell composition and architecture. By incorporating shear flow into this permeable system, the blood flow in sinusoids and interstitial flow in space of Disse are recapitulated. Conventional hepatocyte-based liver-specific functions are enhanced by non-parenchymal cells co-culture and shear flow. Moreover, major immune responses in liver sinusoids, i.e., neutrophil recruitment under lipopolysaccharide (LPS) stimulation, are replicated, indicating that all types of hepatic cells contribute to this process. Thus, this liver chip provides a new in vitro model to investigate the short-duration cellular interactions under a microenvironment mimicking the physiological composition and architecture of liver organ.

\section{INTRODUCTION OF CELL-CELL INTERACTIONS IN LIVER SINUSOIDAL MICROENVIRONMENTS}

\subsection{ARCHITECTURE AND CELL COMPOSITION OF THE LIVER SINUSOIDS}

The liver is the largest organ in the body and has an extraordinary spectrum of functions, including the synthesis or metabolism of carbohydrates, proteins and lipids, the clearance of toxins and pathogens, and the regulation of immune responses. Liver has a dual blood supply system, $25 \%$ from the hepatic artery and $75 \%$ from the portal vein (Vollmar \& Menger, 2009). The hepatic artery branches into arterioles and the portal vein branches into venules. Together with bile duct, the portal triads serve as the functional units of the liver, the liver lobules (Fig. 1). In these polygonal liver lobules, the portal vein venules continue branching to form very specialized capillaries, the liver sinusoids. Main liver sinusoids run between lobule cords over a length of $250 \mu \mathrm{m}$ and a diameter of 7-15 $\mu \mathrm{m}$ (Vollmar \& Menger, 2009). This unique vascular structure could slow the flow of blood, maximizing mass transfer between the circulating blood and the surrounding hepatocytes or HCs (Hu, Lü, Feng, \& Long, 2017). As this sinusoidal endothelial layer does not have an organized basement membrane, a gap called the space of Disse is formed between the endothelium and hepatic plate. In addition, many non-parenchymal cells of the liver are located in the sinusoids and space of Disse (Fig. 1), allowing the efficient immune surveillance and clearance, as well as other metabolic functions.

The liver organ consists of different cell types. Basically, these cells can be classified as parenchymal cells (HCs) and non-parenchymal cells (NPC). In liver sinusoids, there are at least four major types of hepatic cells, that is, hepatocytes, liver sinusoidal endothelial cells (LSECs), Kupffer cells (KCs), and hepatic stellate cells (HSCs). 


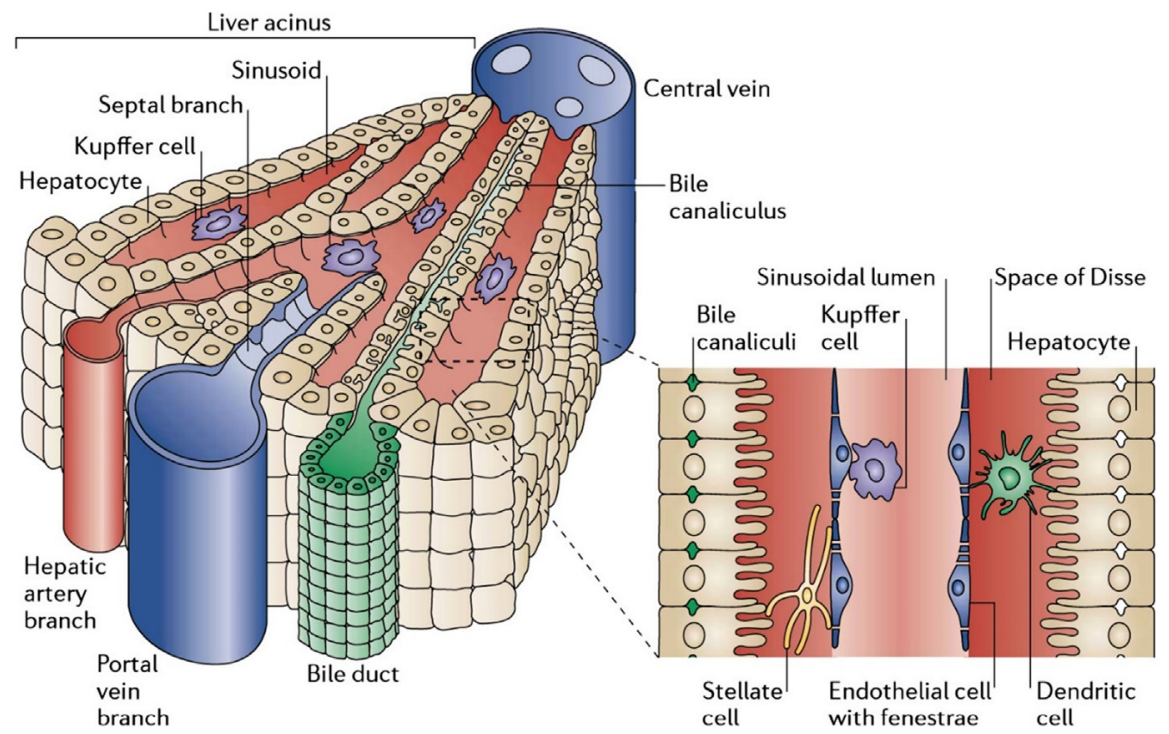

\section{FIG. 1}

Structures of liver lobule and liver sinusoids. Hepatocytes are aligned radially to form the liver plate along with the sinusoids. The portal veins and hepatic artery branches terminate in the sinusoids, draining blood into the sinusoids and through the acinus to the central vein. The sinusoids are lined by fenestrated liver sinusoidal endothelial cells with Kupffer cells interspersed onto the endothelium. Between the liver plate and the sinusoids is the space of Disse, containing extracellular matrix components and hepatic stellate cells.

Reproduced by the permission from Adams, D. H., \& Eksteen, B. (2006). Aberrant homing of mucosal T cells and extra-intestinal manifestations of inflammatory bowel disease. Nature Reviews in Immunology, 6, 244-251.

The hepatocytes account for approximately $80 \%$ of the liver mass, and are the building blocks of the liver lobule. They are distributed radially with sinusoids separating the plate of hepatocytes. These hepatocytes are polygonal and, infrequently binucleated, with a diameter of 20-30 $\mu \mathrm{m}$ (Marra \& Parola, 2011) (Fig. 2A). Hepatocytes are structurally and functionally polarized, characterized by three surface domains: (1) the basal or sinusoidal domain, showing short microvilli and involved in the exchange of molecules with sinusoidal blood; (2) the lateral domain, represented by junctional complexes between adjacent hepatocytes; and (3) the apical or canalicular domain. Hepatocytes serve as the key hepatic effector cells, performing most of the liver functions including synthesis, metabolism, and detoxification.

LSECs are the second most abundant cell type in the liver (approximately $50 \%$ of NPCs) and positioned between the blood and the hepatocytes. LSECs are highly specialized endothelial cells without basement membrane and diaphragm, forming the unique phenotype of fenestration (Fig. 2B) that was first described by Dr. Eddie Wisse in the early 1970s as the fenestrations (Latin fenestrae: "window"). The fenestrae are transcellular pores with a diameter of $150-175 \mathrm{~nm}$ and grouped into $20-50$ 

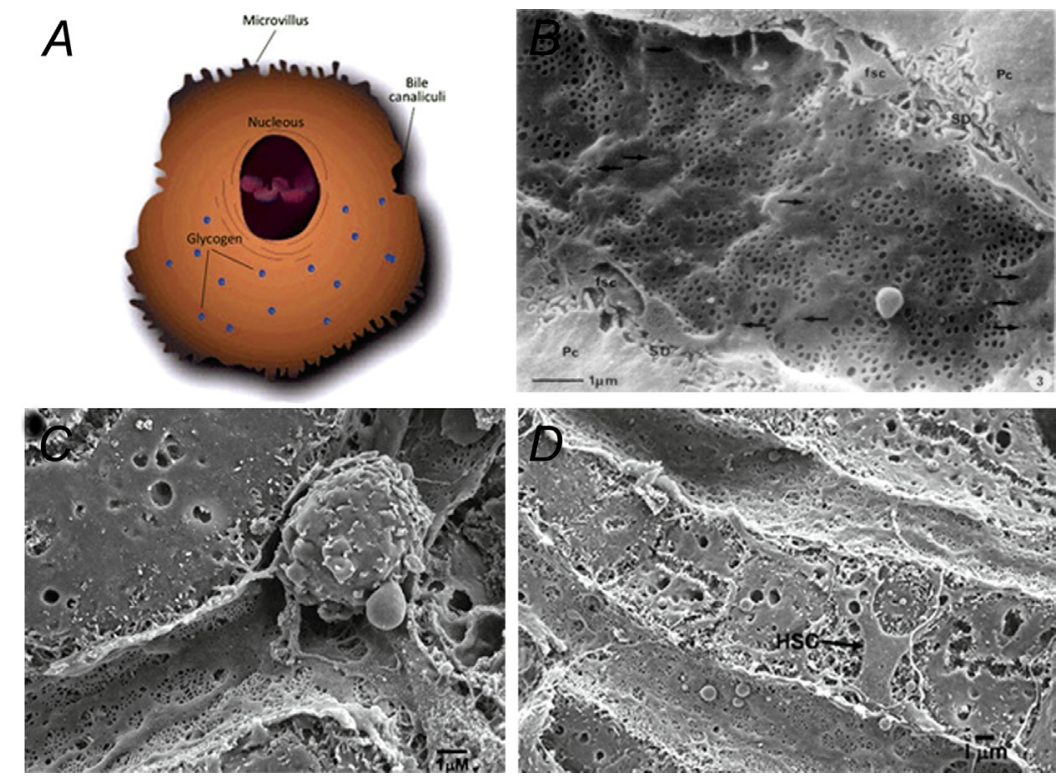

FIG. 2

Four major types of constituent hepatic cells residing in liver sinusoids. (A) Hepatocytes are polarized with three surface domains: the basal or sinusoidal domain, the lateral domain, and the apical or canaliculus domain. Hepatocyte is enriched with smooth endoplasmic reticulum, which correlates with its protein synthesis function. (B) Lining along hepatic sinusoid are the unique endothelial cells with the typical fenestrations integrated as clusters and forming the so-called sieve plates (arrows). (C) A Kupffer cell residing in the sinusoid of the liver. (D) A hepatic stellate cell settling in the space of Disse and spanning across two sinusoids.

Reproduced by the permission from Soto-Gutierrez, A., Navarro-Alvarez, N., \& Kobayashi, N. (2011). Hepatocytes. In S. P. S. Monga (Ed.), Molecular pathology of liver diseases (pp. 17-26). New York, NY: Springer Science+Business Media, LLC; Wisse, E., De Zanger, R. B., Charels, K., Van Der Smissen, P., \& McCuskey, R. S.

(1985). The liver sieve: Considerations concerning the structure and function of endothelial fenestrae,

the sinusoidal wall and the space of Disse. Hepatology, 5(4), 683-692; Gandhi, C. R. (2011). Kupffer cells. In S. P. S. Monga (Ed.), Molecular pathology of liver diseases (pp. 81-95). New York, NY: Springer Science+ Business Media, LLC; Gandhi, C. R. (2011). Stellate cells. In S. P. S. Monga (Ed.), Molecular pathology of liver diseases (pp. 53-79). New York, NY: Springer Science+Business Media, LLC.

fenestrae to form "sieve plates" (Wisse et al., 1996; Wisse, De Zanger, Charels, Van Der Smissen, \& McCuskey, 1985). The fenestrations permit the passive transport of solutes, and more importantly, regulate particular trafficking between the blood supply and the underlying hepatocytes. LSECs are the main source of endothelium derived nitric oxide (NO), a key modulator of vascular tone which is produced by endothelial nitric oxide synthase (eNOS) (Marrone, Shah, \& Gracia-Sancho, 2016; Shah et al., 1997). Moreover, LSECs play an important role in liver inflammation and liver injury by expressing a spectrum of adhesion molecules (Geraud et al., 2012). 
KCs are named after the pathologist Carl von Kupffer identified them as resident liver macrophages. KCs constitute around $80 \%$ of all the tissue macrophages of the reticuloendothelial system and about $15 \%$ of all liver cells (Bilzer, Roggel, \& Gerbes, 2006). They are stationary cells located in the vasculature, adherent to LSECs, and directly exposed to the flow of blood (Fig. 2C). KCs are the first macrophage population to encounter gut-derived bacteria, endotoxin (LPS), and antigens. By expressing an array of scavenger receptors, such as CD163, toll-like receptors (TLRs) especially TLR4, complement receptors and Fc receptors, KCs could detect, bind and internalize pathogens and associated molecules (Bilzer et al., 2006; Su, 2002). Furthermore, KCs could be activated to product cytokines and chemokines that alert other components of the immune system to the presence of harmful pathogens. KCs play crucial roles not only in innate immunity but also in adaptive immunity. By expressing MHC I, MHC II and costimulatory molecules, they could serve as antigen presenting cells to activate $\mathrm{T}$ cells, as exemplified in presenting antigens to iNKT cells via CD1d upon the capture of bacteria (Lee et al., 2010).

HSCs were identified by von Kupffer in 1876 , which comprise a minor fraction of total liver cells (5-8\%). HSCs reside in the Disse space between hepatocytes and LSECs, and extend long, dendrite-like pseudopodia that wrap around the sinusoids (Fig. 2D). Under quiescent conditions, the cells have a central role in vitamin A and lipid storage (Jenne \& Kubes, 2013). In chronic liver damage, however, HSCs are transdifferentiated from a quiescent cell to a fibrinogenic, proliferating cell. These transdifferentiated cells lose retinoid and express greater amounts of rough endoplasmic reticulum. Smooth muscle isoform of $\alpha$-actin ( $\alpha$-SMA) represents a reliable marker for the identity of "activated" HSCs (Friedman, 2008).

\subsection{CELLULAR INTERACTIONS IN THE LIVER SINUSOIDS DURING FIBROSIS AND INFLAMMATION}

Due to the complex structure and cellular component, the liver microenvironment can be described as a multidirectional interaction complex (cell-matrix-cell), where each part (cell or matrix) plays a crucial role (Fig. 3). Cellular interactions within the liver is critical for many hepatic processes, including fibrosis and inflammation (Marrone et al., 2016).

In the presence of hepatic injury, LSECs become rapidly dysregulated and start de-differentiation toward a capillarized phenotype. The cells in turn affect the phenotype of their neighboring cells by producing or releasing soluble factors and exerting mechanical tension on collagen matrix. LSEC-derived fibronectin regulates HSC phenotype and promotes their activation (Jarnagin, Rockey, Koteliansky, Wang, \& Bissell, 1994). In early stage of fibrosis, LSECs activate HSCs by generating contractile force via collagen fibers (Liu et al., 2017). In parallel, exogenous hepatic injury induces hepatocyte apoptosis, resulting in activating the NPCs (HSC and KC) by releasing apoptotic bodies (Canbay et al., 2003; Jiang, Mikami, Venugopal, Li, \& Torok, 2009). Thereafter, activated HSCs begin to proliferate, contract and deposit the elevated amount of collagen fibers and extracellular 


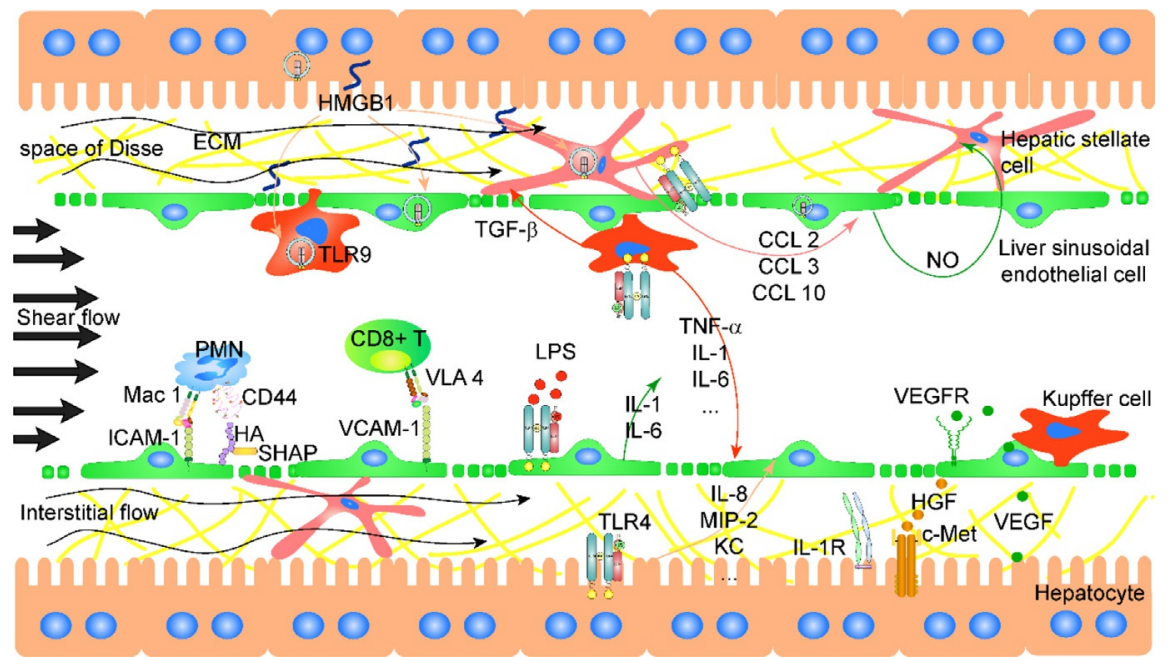

FIG. 3

Cell communication and interplay in liver sinusoids. The sinusoids display their complexity in several aspects: (i) Architecture and cellular composition: multiple types of cells coupled with ECM highly ordered in the liver sinusoids; (ii) Cellular interactions: cross talk of different hepatic cells upon physical contact or soluble factors in many pathophysiological processes such as liver fibrosis and hepatic inflammation; (iii) Mechanical microenvironment: heterogeneous matrix stiffness coupled with the shear flow in sinusoids and the interstitial flow in space of Disse; and (iv) Distinct immune responses: no-rolling recruitment of highly tolerant leukocytes using adhesive molecules different from other tissues.

matrixes (ECMs) in the hepatic parenchyma, contributing to the stiffening of the liver organ and perturbing all the intercellular functions. Meanwhile, collagen accumulation in the Disse space mediates the loss of endothelial fenestrations, aggravating hepatic fibrosis (McGuire, Bissell, Boyles, \& Roll, 1992). Thus, a positive feedback between capillarized LSECs and activated HSCs further augments liver fibrosis.

Hepatic cells interactions also play an important role in liver inflammation (cf. Fig. 3). Facing the challenges of the antigen-rich blood from gastrointestinal tract, $\mathrm{KC}$ is the first exposed as the sentinel in liver sinusoids. Upon LPS stimuli, KCs could clear partially the pathogens, become activated via TLR4 signaling pathway involving MyD88 and NF- $\mathrm{KB}$, and then produce pro-inflammatory cytokines (i.e., TNF- $\alpha$, IL-1, IL-6, IL-17 and IL-18), anti-inflammatory cytokines (i.e., IL-4, IL-10, and IL-13) (Luster, Germolec, Yoshida, Kayama, \& Thompson, 1994; Vollmar \& Menger, 2009) and TGF- $\beta$ to promote HSC activation (Nieto, 2006; Rivera et al., 2007). HSCs could also be activated via TLR4 signaling pathway 
involving JNK and NF-אB to produce CXCL2, CCL3, and CCL10 (Seki et al., 2007), thus upregulating adhesive molecule expression on LSECs to recruit neutrophils by chemokines (Bilzer et al., 2006). LSECs could also be activated to release IL-1 and IL-6, as hepatocytes release IL-8, CXCL1, and MIP-2. After being injured, necrotic and apoptotic hepatocytes could release endogenous ligands such as highmobility group box 1 (HMGB1) and nucleic acids, thus increasing cytokine secretion of NPCs via TLR9-dependent mechanisms and enhancing neutrophil recruitment by regulating adhesive molecule expression on LSECs (Bamboat et al., 2010). All these cytokines and inflammatory mediators contribute to the multistep process of neutrophil recruitment to injured hepatic cells. It should also be pointed out that different hepatic cells have distinct contributions to neutrophil recruitment and the underlying mechanisms are required to dissect elaborately (Edwards, Lalor, Nash, Rainger, \& Adams, 2005; Thornton, Strieter, Lindley, Baggiolini, \& Kunkel, 1990; Yang et al., 2017).

\subsection{SINUSOIDAL MECHANICAL MICROENVIRONMENTS}

The mechanical microenvironment of liver sinusoids is also very sophisticated. Due to the honeycomb vascular structure of sinusoids, the blood flow in the sinusoids is rather slow (Hu et al., 2017). Indeed, the flow velocity is estimated to be $407-451 \mu \mathrm{m} / \mathrm{s}$ in the main sinusoids, significantly slower than the blood flow velocity in the conventional capillaries (500-1000 $\mu \mathrm{m} / \mathrm{s})$ (Oda, Yokomori, \& Han, 2003). As the sinusoidal endothelium is fenestrated, the blood is able to penetrate via the fenestrations and then flow into the Disse space. The interstitial flow is difficult to measure since the Disse space is quite small and discontinuous. This slow interstitial flow dynamics in the sinusoids not only enhances the nutrient exchange and increases the exposure duration of pathogen, but it also affects hepatic cellular functions through fluidic shear stress.

The rigidity of ECM also has a tremendous effect on cellular functions, including cell proliferation and motility. Mechanical measurements of bulk liver tissue of human and rodents by rheometry suggest that the liver organ is quite soft and its stiffness ranges $400-600 \mathrm{~Pa}$ for normal liver and $1.2-1.6 \mathrm{kPa}$ for fibrotic liver (Desai et al., 2016). Using atomic force microscopy, the elaborative, microscale matrix stiffness can be analyzed across liver lobules. The stiffness is around $150 \mathrm{~Pa}$ in normal liver and increased to $1-6 \mathrm{kPa}$ in the areas near fibrillar collagen deposition in fibrotic liver. The normal liver is mechanically heterogeneous, as the stiffness in the periportal zones is slightly higher with greater variability compared to the pericentral zones. As a result, those residing hepatic cells can sense such mechanical signals presented in their microenvironment and activate their downstream intracellular signaling pathways, involving integrin clustering and activation of focal adhesion kinase, Rho/Rho-associated protein kinase, or extracellular signalregulated kinase. 


\subsection{IN VITRO MODELS OF THE LIVER SINUSOIDS}

To better mimic the key features of liver sinusoids, an in vitro model should account, at least, for the following three aspects, the sinusoidal structure, the cellular composition, and the mechanical microenvironment.

In recent decades, there have been multiple in vitro models attempting to recapitulate the liver sinusoids from microscale ultrastructure to tissue level. At ultrastructure level, an individual liver sinusoid is mimicked with a set of parallel channels with a width of $2 \mu \mathrm{m}$ and a height of $1 \mu \mathrm{m}$ between hepatocytes and culture medium (Lee, Hung, \& Lee, 2007) (Fig. 4A). This microfluidic device possesses the mass transport feature of acinus, which, on one hand, guarantees the nutrient transport by convective flow through the endothelial barrier, and, on the other hand, protects the hepatocytes from shear flow by high resistance of the barrier. Further studies are conducted to build up the liver sinusoid models with tissue level functions by considering its architecture, co-culture and fluidic perfusion (Dash et al., 2013). Specifically, primary rat hepatocytes and bovine aortic endothelial cells are separated by a thin matrigel layer or a porous membrane and one or two channels are linked to a continuous flow system. Both co-culture and fluid flow increase the urea secretion, resulting in that this liver model could support longterm primary hepatocyte culture and hepatitis B virus replication (Kang et al., 2015) (Fig. 4B). Moreover, multiple types of cells need to be considered in the liver model due to the complex cellular composition in liver sinusoids. For example, primary human hepatocytes, endothelial cell line, EA.hy926, and macrophage cell line, U937, along with the three-dimensional (3D) dispersal of stellate cell line, LX-2, are arranged in two flow channel separated by a porous membrane (Prodanov et al., 2016) (Fig. 4C). Using this device, it is indicated that the liver sinusoids can be maintained for 28 days, and the flow could enhance albumin and urea synthesis. However, the effects of co-culture are not discussed explicitly in this model. Thus, an alternative liver model that is constructed with HepaRG cells, human umbilical cord vein endothelial cells (HUVECs), macrophages differentiated from peripheral blood mononuclear cells (PBMCs), and LX-2 looks more reasonable in testing the effects of shear flow and co-culture (Rennert et al., 2015) (Fig. 4D). Albumin and urea secretion are increased by co-culture and further by shear flow. The flow also up-regulates the cytochrome enzyme CYP3A4, increases the MRP-2 expression and functions, and enhances the formation of microvilli, where the up-regulation of CYP3A4 is contributed distinctly by different cells. These studies have shown that the in vitro liver models with co-culture and shear flow are capable of replicating multiple morphological and functional characteristics, and are promising to investigate the functions and responses of the liver sinusoids. However, the liver is a complex organ with multiple cells working together and implementing various functions. The importance of NPCs and their contributions to the overall hepatic responses and drug toxicity are becoming much highlighted (Bale, Geerts, Jindal, \& Yarmush, 2016). Except of hepatocyte-based functions, the functionality incorporated with NPCs should also be addressed. Isolation of purified NPCs and controllable incorporation of these cells into an in vitro model are essential for development of better liver sinusoid chips. 
A

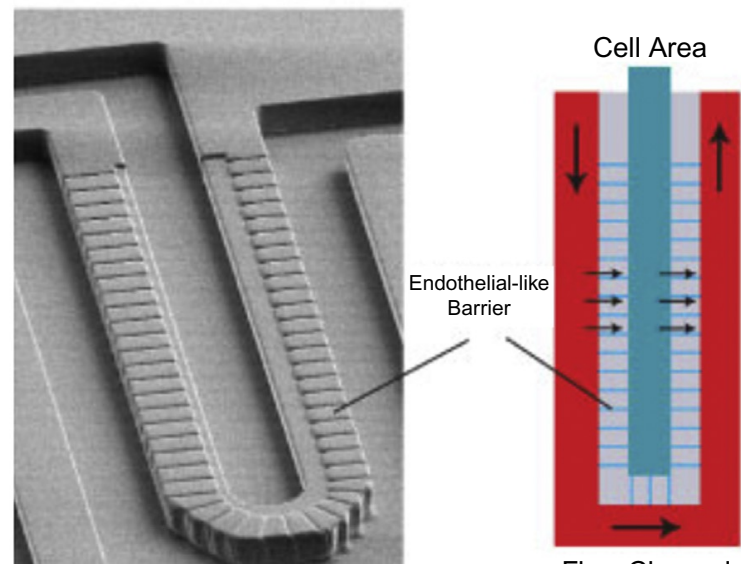

Flow Channel

C

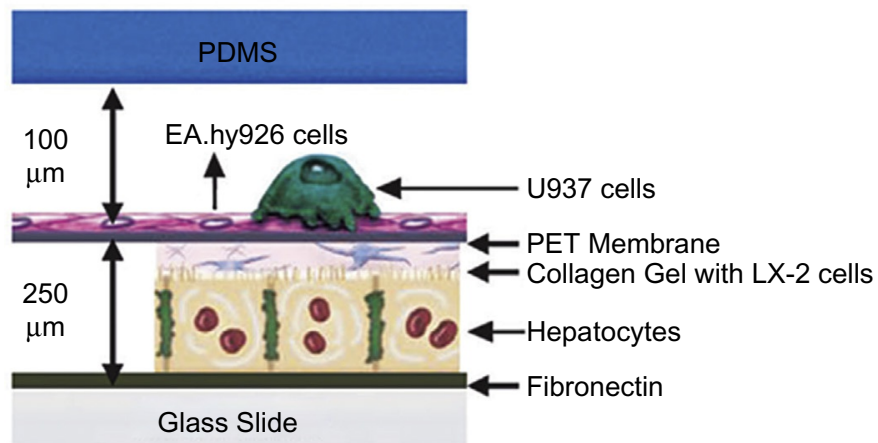

B

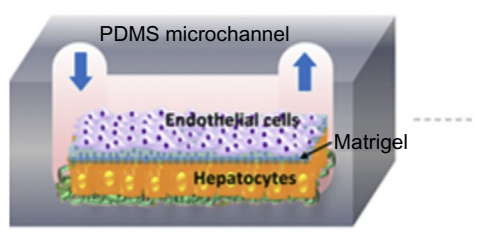

Single channel configuration

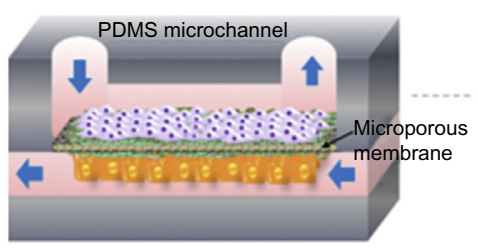

Dual channel configuration

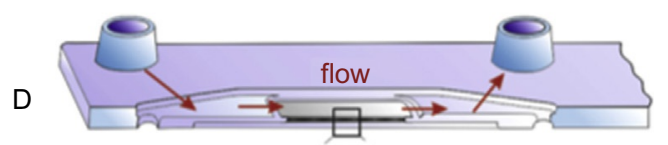

vascular laye human primary

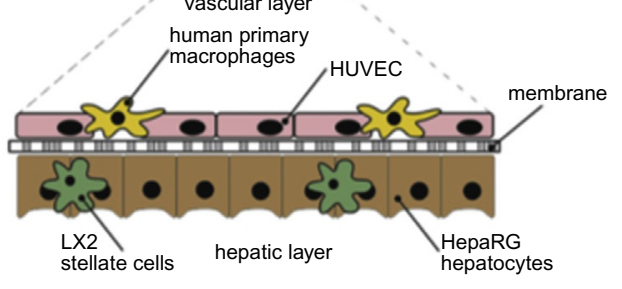

\section{FIG. 4}

Microengineered techniques for developing in vitro liver sinusoid models. (A) A single liver sinusoid with an endothelial-like barrier. (B) A co-culture microfluidic system with primary rat hepatocytes and bovine aortic endothelial cells separated by either a thin matrigel layer or a porous membrane, forming one or two channels. (C) A long-term liver sinusoid model using primary human hepatocytes, endothelial cell line EA.hy926, and macrophage cell line U937 along with the 3D dispersed stellate cell line LX-2 for testing the effects of shear flow alone. (D) A liver model constructed with HepaRG cells, HUVECs, PBMCs and LX-2 for specifying the effects of shear flow and co-culture.

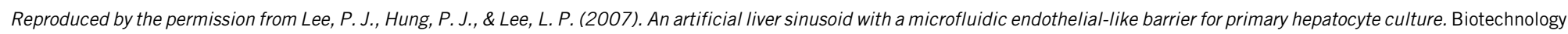
and Bioengineering, 97(5), 1340-1346. doi:10.1002/bit.21360; Kang, Y. B., Sodunke, T. R., Lamontagne, J., Cirillo, J., Rajiv, C., Bouchard, M. J., et al. (2015). Liver sinusoid on

a chip: Long-term layered co-culture of primary rat hepatocytes and endothelial cells in microfluidic platforms. Biotechnology and Bioengineering, 112(12), 2571-2582. https://doi.org/

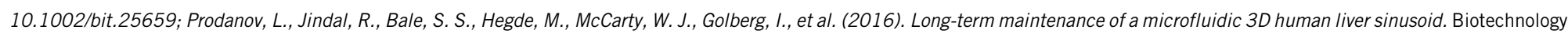

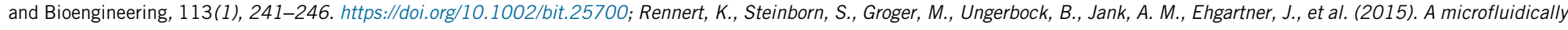
perfused three dimensional human liver model. Biomaterials, 71, 119-131. https://doi.org/10.1016/j.biomaterials.2015.08.043. 
Here we not only fabricate a dual channel permeable system using microengineering techniques but also isolate primary hepatocytes, LSECs, KCs and HSCs from mice (Liu et al., 2011). By integrating these hepatics cells into the microfluidic device, multiple functions and responses of the liver sinusoids are replicated (Du et al., 2017).

\section{CONSTRUCTION OF THE LIVER SINUSOID CHIP}

\subsection{MICROFLUIDIC DEVICE FABRICATION}

Soft lithography techniques are used to fabricate the microfluidic devices. A simplified schematic flow chart is shown in Fig. 5. The main flowing zones of both upper and lower channels are equally sized in $H \times W \times L=100 \mu \mathrm{m} \times 1 \mathrm{~mm} \times 15 \mathrm{~mm}$. The protocol is briefly described as follows:

1. Mix the PDMS (Dow Corning, MI) silicone elastomer base and curing agent completely in a weight ratio of 9:1 (base:curing agent).

2. Degas the PDMS mixture by a vacuum desiccator (Yilibotong Company, China).

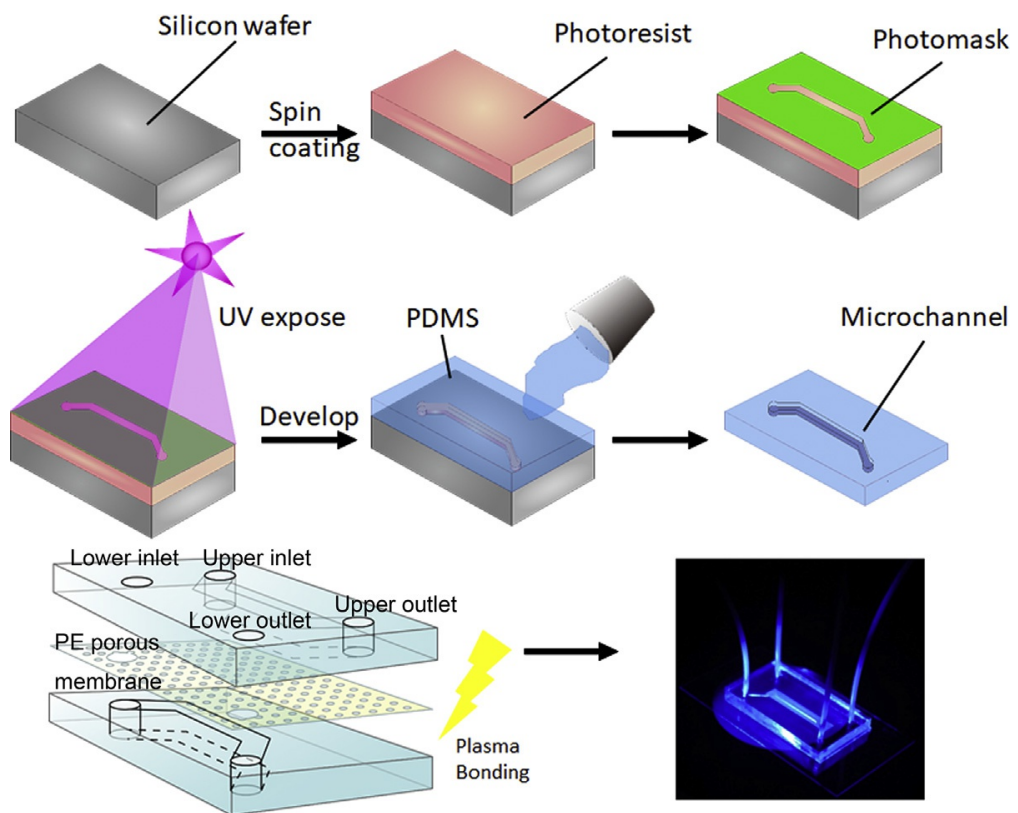

FIG. 5

Fabrication of the microfluidic device. Fabricate the SU-8 mold by lithography technique. Make PDMS layers with the SU-8 mold and bond the two PDMS layers and the porous PE membrane by plasma sputtering. 
3. Pour the degassed PDMS onto the SU-8 mold (Capital Bio Corporation, China).

4. Degas again with the vacuum desiccator.

5. Cure the PDMS gel fully in an oven at $85^{\circ} \mathrm{C}$ for $1 \mathrm{~h}$.

6. Peel the PDMS gel off carefully from the mold.

7. Cut the gel into bricks with equal size.

8. Punch the holes at inlet and outlet position with a hole puncher.

9. Cut the $0.4 \mu \mathrm{m}$-diameter pore-sized polyester (PE) membrane into the rectangle pieces with enough size to cover the overlapped channel area.

10. Plasma treat the contact surfaces between upper and lower PDMS layers with Plasma Sputtering Pump (Yilibotong, China) for $1 \mathrm{~min}$.

11. Align the two layers and PE membrane carefully, and press gently and uniformly to ensure that they are fully bonded.

12. UV the integrated chip for $30 \mathrm{~min}$ to sterilize.

13. Coat both the channels with $100 \mu \mathrm{g} / \mathrm{mL}$ collagen $\mathrm{I}$ at $4{ }^{\circ} \mathrm{C}$ overnight to ensure cell attachment before use.

\subsection{PRIMARY HEPATIC CELL ISOLATION}

6-8 week C57BL/6 mice (Vital River Laboratories, China) are used to isolate primary murine hepatic cells. All animal experiments are approved by the Animal and Medicine Ethical Committee of the Institute of Mechanics, Chinese Academy of Sciences. A modified two-step collagenase digestion protocol is applied to digest liver tissue (Braet et al., 1994; Liu et al., 2011) (Fig. 6), as briefed below.

1. Prewarm the $\mathrm{Ca}^{2+}$-free Gey's balanced salt solution $(\mathrm{NaCl} 8.0 \mathrm{~g}, \mathrm{KCl} 0.4 \mathrm{~g}$, $\mathrm{NaH}_{2} \mathrm{PO}_{4} 0.0926 \mathrm{~g}, \mathrm{Na}_{2} \mathrm{HPO}_{4} 0.287 \mathrm{~g}$, HEPES $2.38 \mathrm{~g}, \mathrm{NaHCO}_{3} 0.35 \mathrm{~g}$, glucose $0.901 \mathrm{~g}$, heparin $0.1 \mathrm{~g}$, EGTA $0.19 \mathrm{~g}$ in $1 \mathrm{~L} \mathrm{DI}$ water, $\mathrm{pH} 7.4,0.22 \mu \mathrm{m}$ filtered) and collagenase IV solution $\left(\mathrm{CaCl}_{2} 0.022 \mathrm{~g}\right.$, FBS $1 \mathrm{~mL}$, collagenase IV $0.025 \mathrm{~g}$ in $50 \mathrm{~mL}$ DMEM, $0.22 \mu \mathrm{m}$ filtered) to $37^{\circ} \mathrm{C}$ before surgery.

2. Clear the bubbles in the perfusion system (this step is crucial to ensure that the mice liver is well perfused).

3. Anesthetize the mice by intraperitoneal injection with Nembutal $(30 \mathrm{mg} / \mathrm{kg})$. Check if the mice is completely unconscious by squeezing toe without reaction.

4. Place the animal in a supine position with all limbs fixed. Clean the abdomen with $75 \%$ ethanol.

5. Expose the liver and portal vein by a ventral midline incision.

6. Insert a $22 \mathrm{G}$ catheter into the portal vein.

7. Start the perfusion of the $\mathrm{Ca}^{2+}$-free Gey's balanced salt solution at a rate of $5 \mathrm{~mL} / \mathrm{min}$ for $5 \mathrm{~min}$.

8. Switch to the collagenase IV solution at a rate of $5 \mathrm{~mL} / \mathrm{min}$ for additional $5 \mathrm{~min}$.

9. Excise carefully the liver and transfer into high glucose DMEM medium (Hyclone, UT) in a sterile Petri dish.

10. Mince the liver into small pieces, and pipe the medium several times. 

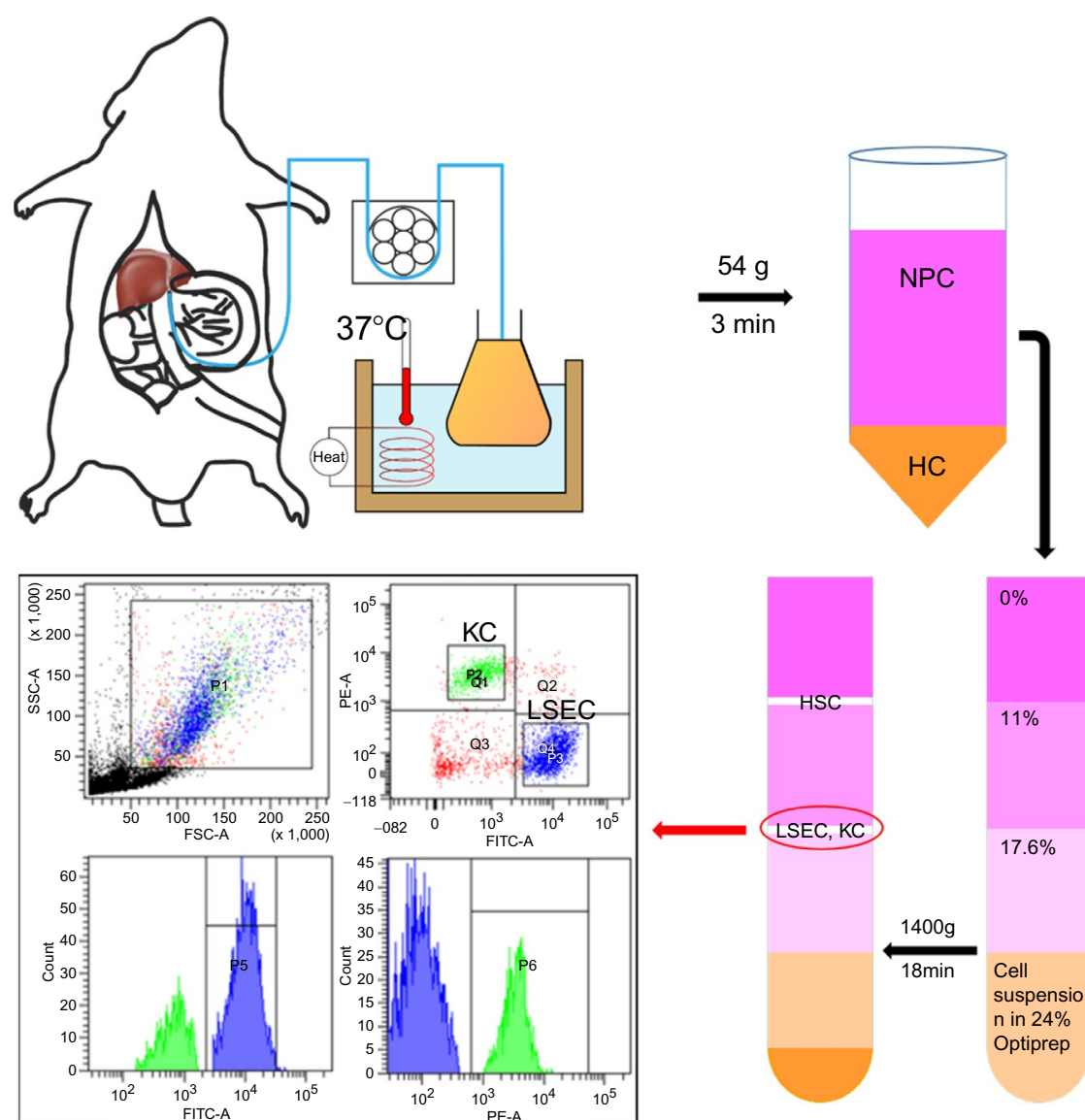

FIG. 6

Two-step perfusion, density gradient centrifugation and flow cytometry sorting for hepatic cells isolation. The whole liver is digested by perfusion with $\mathrm{Ca}^{2+}$-free Gey's balanced salt solution followed by collagenase. HCs are harvest from the low speed centrifugation. HSCs can be harvest from the density gradient centrifugation. The mixture of LSECs and $\mathrm{KCs}$ is further purified using flow cytometry sorting. After removing the cell debris, LSECs $\left(\mathrm{CD} 146^{+}, \mathrm{F} 4 / 80^{-}\right)$and $\mathrm{KCs}\left(\mathrm{CD} 146^{-}, \mathrm{F} 4 / 80^{+}\right)$are collected respectively.

11. Filter the homogenate through a cell strainer $(200 \mu \mathrm{m}$ in diameter $)$ to remove undigested tissue fragments.

12. Centrifuge the cell suspension at $54 \times g$ at $4{ }^{\circ} \mathrm{C}$ for $3 \mathrm{~min}$.

For hepatocytes

13. Collect the packed cell, resuspend in the high glucose DMEM and centrifuge to collect the pellet for three times at $54 \times g$ at $4{ }^{\circ} \mathrm{C}$ for $3 \mathrm{~min}$ each. 
14. Resuspend the hepatocytes in culture medium (high glucose DMEM supplemented with $10 \%$ FBS, $100 \mu \mathrm{g} / \mathrm{mL}$ streptomycin and $100 \mathrm{U} / \mathrm{mL}$ penicillin) into a final concentration of $5 \times 10^{5} / \mathrm{mL}$ and use immediately.

\section{For NPCs}

15. Centrifuge the supernatant obtained from the Step 12 at $500 \times g$ for $8 \mathrm{~min}$.

16. Resuspend the pellet with $3 \mathrm{~mL}$ of $24 \%$ Optiprep solution (Axis-Shield, Norway).

17. Load with $17.6 \%, 11.7 \%$ Optiprep solution and $3 \mathrm{~mL}$ DMEM orderly in a $15 \mathrm{~mL}$ conical tube.

18. Centrifuge at $1400 \times g$ for $18 \mathrm{~min}$.

\section{For HSCs}

19. Collect the layer between DMEM and $11.7 \%$ Optiprep, dilute with twice volume of Dulbecco's phosphate-buffered saline (DPBS, Hyclone, UT), and centrifuge at $1400 \times g$ for $8 \mathrm{~min}$.

20. Resuspend the cells in culture medium into a final concentration of $1 \times 10^{6} / \mathrm{mL}$ and use immediately.

\section{For LSECs and KCs}

21. Collect the layer between $11.7 \%$ and the $17.6 \%$ Optiprep, dilute with twice volume of DPBS, and centrifuge at $1400 \times g$ for $8 \mathrm{~min}$.

22. Resuspend the pellet in $90 \mu \mathrm{L}$ of DPBS per $10^{8}$ cells.

23. Incubate with $5 \mu \mathrm{L}$ FITC-conjugated rat-anti-mouse CD146 mAbs (Miltenyi Biotec, Germany) and $5 \mu \mathrm{L}$ PE-conjugated recombinant human-anti-mouse F4/80 mAbs (Miltenyi Biotec, Germany) at $4{ }^{\circ} \mathrm{C}$ in dark for $15 \mathrm{~min}$.

24. Wash twice with DPBS, and resuspend into a final concentration of $10^{8} / \mathrm{mL}$ for flow cytometry sorting with FACS Aria III (BD Biosciences, NJ).

25. Isolate the LSECs by $\mathrm{CD} 146^{+} \mathrm{F} 4 / 80^{-}$gating and the $\mathrm{KCs}$ by $\mathrm{CD} 146^{-} \mathrm{F} 4 / 80^{+}$ gating.

26. Mix the sorted LSECs and KCs with $\sim 100 \%$ purity in 2:1 ratio as the in vivo proportion, resuspended in culture medium into a final concentration of $5 \times 10^{6} / \mathrm{mL}$, and use immediately.

\subsection{IDENTIFICATION OF ISOLATED HEPATIC CELLS}

The type-specific morphology and biomarker expression of the four cell types are first tested in conventional dishes. It is found that these specific features are well presented and maintained. That is, glycogen-containing HCs display a polygonal shape with double nucleus and clear edges, CD146-identified LSECs form a monolayer presenting a cobblestone shape, F4/80-identified KCs yield a polymorphic shape, and glial fibrillary acidic protein (GFAP)-specific HSCs present a stellate-like shape (Fig. 7). 

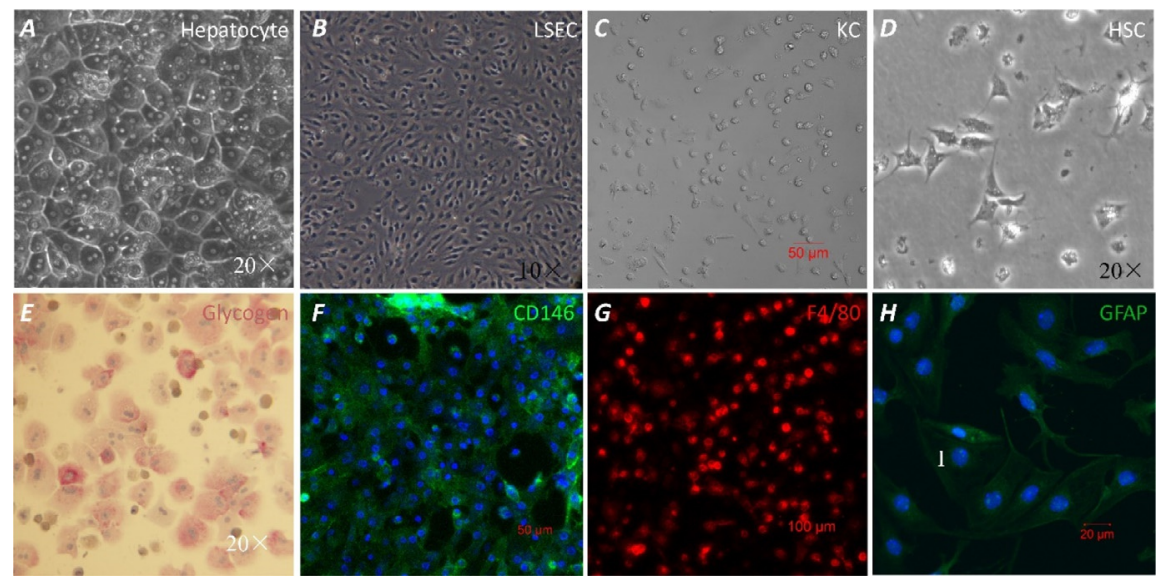

\section{FIG. 7}

Characterization of four types of primary hepatic cells from mouse liver. Hepatocytes display a polygonal shape with double nucleus and clear edges (A) and almost all hepatocytes contain glycogen as stained in purple (E). LSECs form a monolayer presenting a cobblestone shape (B) and are identified with CD146 as stained in green (F). KCs yield a polymorphic shape (C) and are defined with F4/80 as stained in red (G). HSCs have a stellate-like shape (D) and are marked with GFAP as stained in green $(\mathrm{H})$.

\section{Glycogen synthesis of hepatocytes}

Glycogen synthesis of hepatocytes is tested by a commercial kit using a Periodic Acid-Schiff stain (Nanjing Jiancheng Bioengineering Institute, Nanjing, China) according to the manufacturer's instructions. Briefly,

1. Dilute the powders of periodic acid and Schiff's reagent in their respective diluting solutions and then balance at room temperature (RT) for $20 \mathrm{~min}$.

2. Fix the cells with $95 \%$ ethanol for $2-5 \mathrm{~min}$ and wash for $1 \mathrm{~min}$.

3. Add the periodic acid working solution to the fixed cells and incubate for $15 \mathrm{~min}$.

4. Rinse with water for $3-5 \mathrm{~min}$.

5. Add the Schiff's working solution and incubate for $15 \mathrm{~min}$.

6. Wash for $30-60 \mathrm{~s}$.

7. Add the hematoxylin staining solution, and incubate for $20-30 \mathrm{~s}$, and flush out the solution.

\section{Immunofluorescent staining (IF)}

1. Fix the cells by $4 \%$ paraformaldehyde (PFA) for $15 \mathrm{~min}$ at RT.

2. Wash three times with DPBS.

3. Solubilize the cells with $0.1 \%$ Triton $\mathrm{X}-100$ for $10 \mathrm{~min}$ at RT.

4. Wash three times with DPBS.

5. Incubate the cells in IF blocking buffer (1\% BSA in DPBS) for $1 \mathrm{~h}$ at $37^{\circ} \mathrm{C}$. 
6. LSECs and KCs are incubated with FITC-conjugated rat-anti-mouse CD146 and PE-conjugated recombinant human-anti-mouse F4/80 mAbs, respectively, at $4{ }^{\circ} \mathrm{C}$ in dark for $15 \mathrm{~min}$. HSCs are incubated with rabbit polyclonal anti-GFAP antibodies (Abcam, UK) at $4{ }^{\circ} \mathrm{C}$ overnight, followed by FITC-conjugated donkey anti-rabbit secondary antibodies (Abcam, UK). All antibodies are diluted to $5 \mu \mathrm{g} / \mathrm{mL}$ in blocking buffer.

7. Wash three times with DPBS and collect the images of stained cells using a Zeiss LSM710 confocal microscope system (Zeiss, Oberkochen, Germany) with a $20 \times$ oil immersion objective.

\subsection{CHIP ASSEMBLING AND CHARACTERIZATION}

\section{Chips assembling}

1. Introduce $6 \mu \mathrm{L}$ of HSC suspension $\left(1 \times 10^{6} / \mathrm{mL}\right)$ into the lower channel.

2. Block the inlets and outlets of the upper channel rapidly, and invert the chip.

3. Allow the HSCs attached onto the basolateral surface of the porous PE membrane after being incubated at $37^{\circ} \mathrm{C}$ in $5 \% \mathrm{CO}_{2}$ for $1 \mathrm{~h}$.

4. Inject $6 \mu \mathrm{L}$ of mixed suspension $\left(5 \times 10^{6} / \mathrm{mL}\right)$ of LSECs and KCs (2:1 ratio) into the upper channel, and $6 \mu \mathrm{L}$ of $\mathrm{HCs}\left(5 \times 10^{5} / \mathrm{mL}\right)$ into the lower channel.

5. Incubate the chip at $37^{\circ} \mathrm{C}$ in $5 \% \mathrm{CO}_{2}$ for $6 \mathrm{~h}$.

6. Wash the unattached cells away from both the channels.

7. Block the inlet and outlet of the lower channel.

8. Introduce the medium flow to the upper channel by the syringe pump at 0.1 or $0.5 \mathrm{dyn} / \mathrm{cm}^{2}$.

9. Culture the chip in the incubator at $37^{\circ} \mathrm{C}$ in $5 \% \mathrm{CO}_{2}$ for $24 \mathrm{~h}$.

10. Collect the supernatants at $24 \mathrm{~h}$ in the presence or absence of shear flow for the tests of protein production and urea secretion.

\section{Biological characterization}

The cellular features of the 3D liver sinusoid chip is characterized by immunostaining of the hepatic cells.

1. Stain the HSCs before seeding with $1 \mu \mathrm{M}$ CellTracker ${ }^{\mathrm{TM}}$ Green (ThermoFisher, MA) at $37^{\circ} \mathrm{C}$ for $15 \mathrm{~min}$.

2. Fix the cells after $24 \mathrm{~h}$ cell culture, by $4 \%$ PFA for $15 \mathrm{~min}$ at RT.

3. Wash three times with DPBS.

4. Permeabilize the cells with $0.1 \%$ Triton $X-100$ for $10 \mathrm{~min}$ at RT.

5. Wash three times with DPBS.

6. Stain the HCs by rabbit anti-E-cadherin mAbs (Cell Signaling Technology, MA) and Alex Fluor 647-conjugated anti-Cytokeratin-pan mAbs (Biolegend, CA) at $4{ }^{\circ} \mathrm{C}$ overnight, followed by FITC-conjugated donkey anti-rabbit secondary antibodies (Abcam, UK). Stain the LSECs by anti-CD146 mAbs, the KCs by anti-F4/80 mAbs and all the cell nuclei with Hoechst 33342.

7. Wash three times with DPBS and collect the images using confocal microscope. 

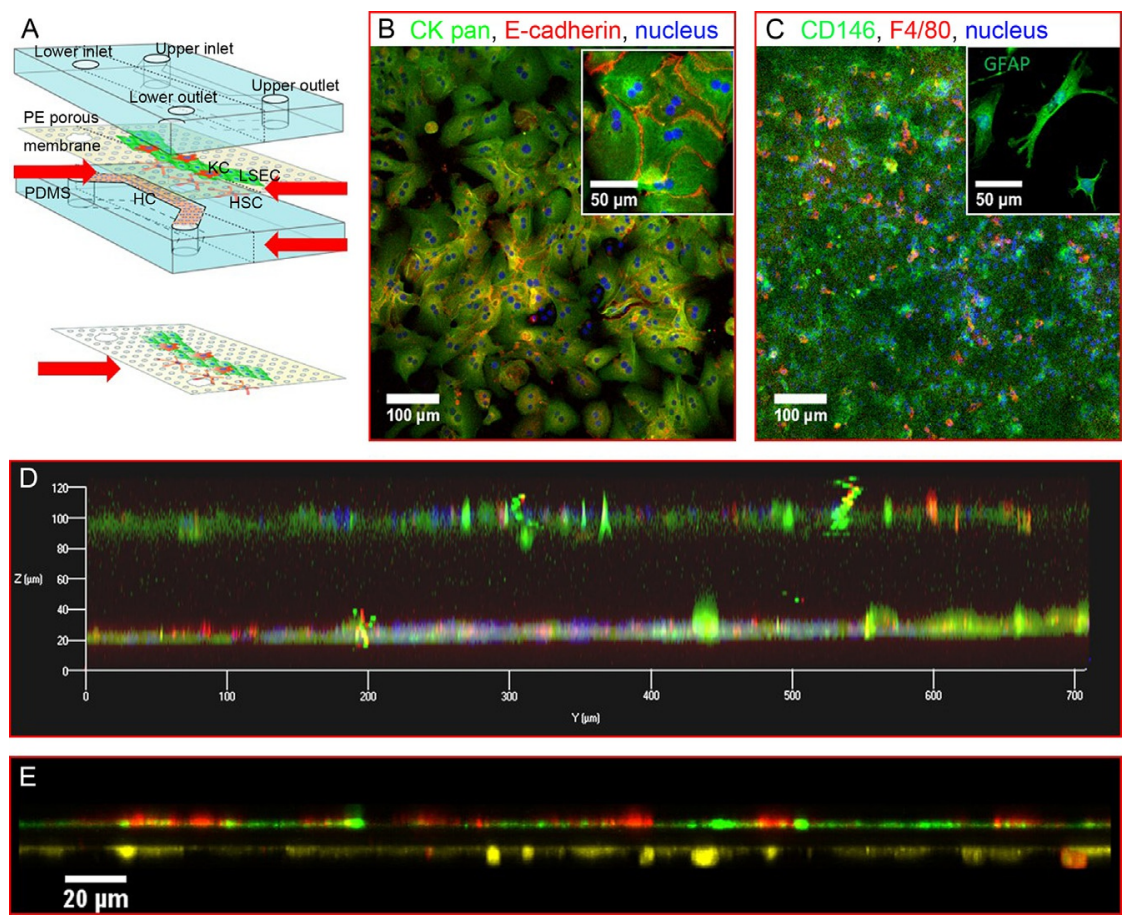

FIG. 8

Assembling and characterization of in vitro 3D liver sinusoidal chip after 1 day. (A) The chip comprises two PDMS chambers of $100 \mu \mathrm{m}$ height and $1 \mathrm{~mm}$ width separated by a $10 \mu \mathrm{m}$ thick PE membrane containing pores with $0.4 \mu \mathrm{m}$ diameter. (B) The bottom layer of HCs stained with E-cadherin (red), CK pan (green) and nucleus (b/ue) are seeded in the lower channel of the chip. Also shown in the inset is a typical high-magnified image. (C) Two layers of cells attached on the porous membrane. LSECs (green; stained with CD146), KCs (red; stained with F4/80) and HSCs (unstained for clarity) are visualized together with nucleus (blue; stained with Hoechst) in the upper channel of the chip. HSCs are stained with GFAP separately (green) and presented in the inset Microfluidic structure. (D) Side view images of the chip illustrate multi-types of the cells in two separated channels.

(E) High-magnified lateral view of sinusoidal endothelium. LSECs (green; stained with $\mathrm{CD} 146$ ) and $\mathrm{KCs}$ (red; stained with F4/80) seeded on apical side of $10 \mu \mathrm{m}$ thick porous membrane, and HSCs (yellow; stained with CellTracker ${ }^{\mathrm{TM}}$ Green) placed on basolateral side.

As indicated by the arrows in the schematic (Fig. 8A), the HCs form an intact monolayer on the lower channel substrate (Fig. 8B). Here HCs present the double nuclei in single cells and are lined by continuous junctional complexes as stained by E-cadherin (inset in Fig. 8B). Within the upper channel, LSECs form a monolayer on the apical side of PE membrane, together with KCs sparsely anchored on top of 
the endothelium-like sentinels (Fig. 8C), and HSCs are scattered on the basolateral side of PE membrane (inset in Fig. 8C). Two channels are integrated well and identified via the side view (Fig. 8D), clearly demonstrating the sinusoidal endothelium and parenchymal plate. A zoomed image around PE membrane indicates that the upper LSEC layer with sparsely distributed KCs and the lower HSC layer are well separated by $10 \mu \mathrm{m}$-distance of the membrane height (Fig. 8E).

Thus, the reconstructed liver sinusoidal chip is able to replicate an in vivo liver sinusoids in cellular composition and architecture.

\section{MECHANICAL MICROENVIRONMENT ANALYSIS}

To better understand the features of fluid flow in the liver sinusoidal chip, a computational flow dynamics (CFD) simulation is done using a model constructed upon the realistic chip geometry (Fig. 9A).

A

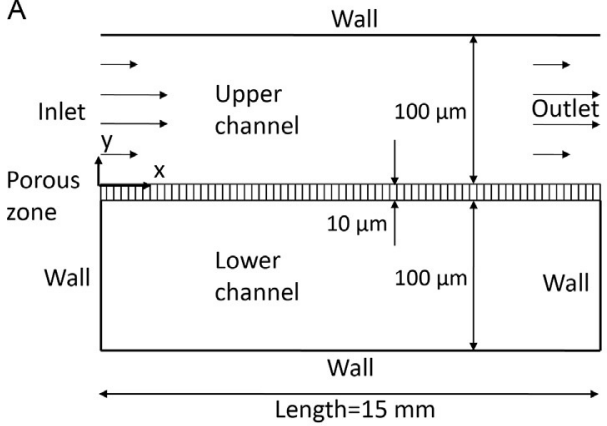

C

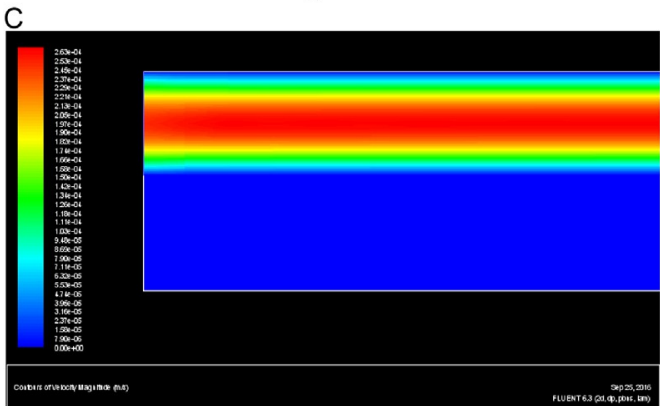

B
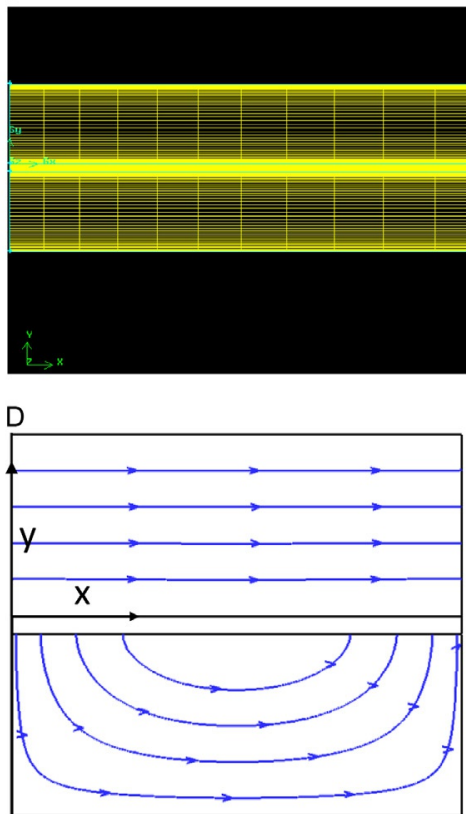

FIG. 9

CFD simulations of fluid flow field in the chip. (A) Geometry of the computational model (not proportional to the actual size). (B) The mesh model created by Gambit. (C) Velocity profile at the left end of the chip. (D) Stream lines inside the chip. 


\subsection{FLUIDIC DYNAMIC MODEL}

CFD model is built by GAMBIT 2.0 (Fig. 9B). The modeling procedure is summarized as flows.

1. Create the frame of the model

(i) Create all vertexes upon the dimension of the model.

(ii) Connect the vertexes into boundary lines.

(iii) Create the faces by specifying lines enclosing a rectangular region.

2. Create the mesh of the model

(i) Create the mesh for the edges.

(ii) Create the mesh for the surfaces with the default settings.

3. Create the boundaries

(i) Set the upper left line as velocity inlet and upper right lines as outflow. Set the porous membrane as a rectangular region, and as fluid by using porous zone model. Set other lines as wall.

The void fraction $\varepsilon$ is obtained from the following equation:

$$
\varepsilon=\frac{\pi d^{2} \rho}{4}
$$

Here $d$ is the pore diameter and $\rho$ is the pore density. As the flow in the chip could be modeled as a laminar flow through a packed bed, the pressure drop is typically proportional to velocity and the constant inertial resistance factor can be considered to be zero. Ignoring convective acceleration and diffusion, the Darcy's Law is satisfied:

$$
\nabla p=-\frac{\mu}{\alpha} \vec{v}
$$

Here $p$ is the pressure, $\mu$ is the viscosity, $\alpha$ is the permeability, and $v$ is the velocity. When modeling laminar flow through a packed bed, Blake-Kozeny equation, a semiempirical correlation, is applicable over a wide range of Reynolds number:

$$
\frac{\Delta p}{L}=\frac{150 \mu}{D_{p}^{2}} \frac{(1-\varepsilon)^{2}}{\varepsilon} v_{\infty}
$$

Here $L$ is the thickness of the packed bed, $D_{p}$ is the mean particle diameter, and $v_{\infty}$ is the velocity in the far field. Comparing the Eqs. (2) and (3), the permeability could be identified as:

$$
\alpha=\frac{D_{p}^{2}}{150} \frac{\varepsilon^{3}}{(1-\varepsilon)^{2}}
$$

As Eq. (4) is a semi-empirical equation and it is not able to determine experimentally the porosity of the PE membrane with LSECs, we first calculate a theoretical permeability by Eq. (4) and then adjust the permeability to $2.5 \times 10^{-16} / \mathrm{m}^{2}$ or $2.5 \times 10^{-17} / \mathrm{m}^{2}$ in the absence or presence of LSECs. These values fit well with those 
velocity profiles measured from PIV tests to represent the $0.4 \mu \mathrm{m}$ porous membrane alone or together with LSECs. For a steady flow, the velocity profile within the parallel flow channel can be obtained analytically, given by a parabolic solution of Poiseuille flow as shown in Eq. (5):

$$
v(y)=6 v y(h-y) h 2 v(y)=\frac{6 \bar{v}(h-y)}{h^{2}}
$$

Here $\bar{\nu}$ is the average velocity, $h$ is the channel height, and $y$ is the $Y$ coordinate along the height direction. Thus, the inlet velocity profile is set as a parabolic distribution by a user defined file (UDF) to achieve faster convergence.

The models built by Gambit can be input into Fluent directly. The running procedure of Porous Zone Model is summarized as follows.

1. Open Fluent 6.3.26 for two-dimensional model with double precision (2ddp).

2. Check the model if Gambit model is correctly built.

3. Scale the grid to millimeter.

4. Select $\mathrm{H}_{2} \mathrm{O}$ from the database.

5. Define the boundary condition.

6. Check the plot in the monitor dialog.

7. Initialize the model.

8. Run the iteration to start the numerical calculation until the program converges.

9. Report the result.

CFD calculation with FLUENT Numerical calculations indicate that the flow is steady and the fluid velocity in the upper channel is much higher than that in the lower channel, presenting a parabolic profile at least in the upper channel (Fig. 9C). Streamlines in the upper channel are parallel to the channel substrate, coming from the inlet and terminating at the outlet (Fig. 9D). By contrast, fluid flow in the lower channel is driven by the flow deriving from the upper channel and across the porous PE membrane, resulting in the symmetric streamlines stemming from the inlet of and terminating at the outlet of the upper channel (Fig. 9D). These data also demonstrate that the fluid is able to penetrate through the porous membrane down to the lower channel near the inlet region and pass through the membrane back to the upper channel around the outlet region. These analyses provide a clue for the flow field inside the chip, which is critical for understanding mass transfer under fluid flow.

\subsection{FLUID FIELD VISUALIZATION}

Particle tracking visualization (PTV) test is conducted for visualization of the flow field and the quantified data are compared with the computations. Since the permeable flow along $Y$ direction is too slow to measure (Fig. 9C), only the $X$ velocity in the chip is measured by visualizing the movement of suspending particles in the chip. Briefly, 
A

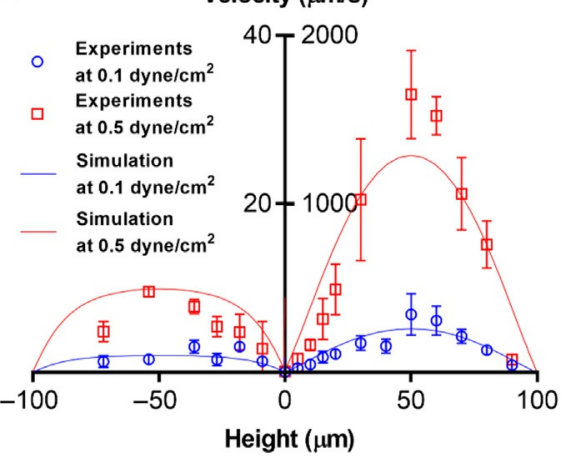

B Velocity at 0.1 dyne $/ \mathrm{cm}^{2}(\mu \mathrm{m} / \mathrm{s})$

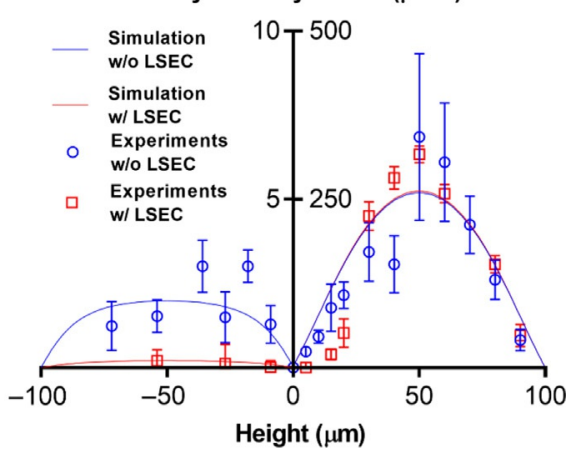

FIG. 10

The PTV test of the velocity profile in the chip. Computational (lines) and measured (points) $X$-direction velocity profiles at the midpoint of the liver chip $(X=7.5 \mu \mathrm{m})$, either at wall shear stress of 0.1 (b/ue) or 0.5 (red) dyn $/ \mathrm{cm}^{2}$ without LSECs (A) or in the presence (red) or absence (b/ue) of LSECs at $0.1 \mathrm{dyn} / \mathrm{cm}^{2}$ (B).

1. Dilute the $1 \mu \mathrm{m}$ diameter carboxylate-modified microspheres (ThermoFisher, MA) labeled with orange fluorescence (540/560) to a concentration of $3.8 \times 10^{7} / \mathrm{mL}$ with water.

2. Add $6 \mu \mathrm{L}$ of the particle solution into the lower channel, and block its inlet and outlet.

3. Introduce the particle solution into the upper channel by the PHD22/2000 syringe pump (Harvard Apparatus, MA).

4. Adjust the height of focus plane through two channels of the chip by Piezo Transducer (Physik Instrumente, Germany).

5. Collect the images by CSU-X1 dual spin disk confocal (Yokogawa, Japan).

In the absence of LSECs, the data present the higher velocity profile at $0.5 \mathrm{dyn} / \mathrm{cm}^{2}$ than that at $0.1 \mathrm{dyn} / \mathrm{cm}^{2}$, both of which all yield a parabolic distribution in either the upper or lower channel and also fit well with the predictions from CFD simulations (Fig. 10A). In the presence of LSECs at a given shear stress of $0.1 \mathrm{dyn} / \mathrm{cm}^{2}$, both the PIV tests and CFD simulations indicate that the parabolic flow inside the chip is maintained with highly-reduced magnitude of flow velocity in the lower channel. The presence of LSECs on the porous membrane blocks a large fraction of membrane pores (Fig. 10B), leading a remarkable decrease of membrane permeability.

\section{LIVER-SPECIFIC FUNCTIONS OF THE SINUSOIDAL CHIP}

\section{ALB, Urea, HGF and VEGF secretion assay}

1. Collect the supernatants after $24 \mathrm{~h}$ from the chip, and centrifuge at $500 \times g$ at $4{ }^{\circ} \mathrm{C}$ for $8 \mathrm{~min}$ to remove cell debris.

2. Store at $-80^{\circ} \mathrm{C}$ before use. 
3. ALB, HGF, and VEGF concentrations are tested by ELISA assay kits for mouse ALB (Bethyl Laboratory, TX), HGF (R\&D, MN), and VEGF (R\&D, MN) in the respective sensitivity of $<1.23 \mathrm{ng} / \mathrm{mL}, 1.33 \mathrm{pg} / \mathrm{mL}$ and $3 \mathrm{pg} / \mathrm{mL}$. Urea concentration is quantified by commercial urea assay kit (Stanbio Laboratory, TX).

\section{Cytochrome P450 (CYP) activity assay}

1. Use the medium supplemented with $200 \mu \mathrm{M}$ phenacetin (for $1 \mathrm{~A} 2$ metabolic test) or $30 \mu \mathrm{M}$ dextromethorphan (for 2D6 test) after hepatic cells are well attached.

2. Collect the supernatants after $24 \mathrm{~h}$ culture from the chip, and centrifuge at $500 \times g$ at $4{ }^{\circ} \mathrm{C}$ for $8 \mathrm{~min}$ to remove cell debris.

3. Store at $-80^{\circ} \mathrm{C}$ before use.

4. Analyze the metabolic products of phenacetin and dextromethorphan, respectively, the acetaminophen and dextrorphan, by LC-MS/MS (Agilent 1200 HPLC, CA and API 4000 mass-spectrometer, CA) at RILD Company

(Shanghai). Substrates and metabolized products used for calibrating curves are commercially purchased from RILD Company.

Compared with those under static condition, the ALB secretion is $78 \%$ increased for HCs alone or $50 \%$ enhanced for HCs co-cultured with NPCs at a shear flow of $0.1 \mathrm{dyn} / \mathrm{cm}^{2}$. Combination of NPC co-culture with flow exposure yields even higher ALB secretion $(112 \%$ or $19 \%$ increased from HCs alone under static condition $(P=0.001)$ or under shear flow), implying the possible cooperative effects of the two factors (Fig. 11A). These results indicate that both co-culture with NPCs and exposure of shear flow enhance ALB secretion independently or cooperatively. By contrast, urea secretion remains similar since no exogenous ammonia is added, no matter whether HCs are co-cultured with NPCs and/or exposed to shear flow (Fig. 11B). No significant difference is found in urea secretion, even though a descending transition is exhibited from HCs alone to HCs co-cultured with NPCs and exposed to shear flow.

Another key feature of liver sinusoids is cytotoxic metabolism by CYP450 in HCs. Here the activities of two typical members of this family, CYP1A2 and CYP2D6, are tested using the constructed liver chip. Shear flow dramatically enhances CYP1A2 (Fig. 11C) and 2D6 (Fig. 11D) activities by 530\% and 628\% when $\mathrm{HCs}$ are cultured alone or by $242 \%$ and $651 \%$ when co-cultured with NPCs, after HCs are pre-treated by respective phenacetin and dextromethorphan. By contrast, co-culture with NPCs has no effects on CYP activities under either static or flow condition. Moreover, combining flow exposure with NPC co-culture presents remarkable increase of CYP1A2 $(277 \% ; P=0.004)$ or 2D6 activity (523\%; $P=0.002$ ). These values are slightly lower than those under shear flow in the absence of NPCs, presumably attributed to mechanical shielding of NPC layers to flow-enhanced CYP metabolism of HCs.

HCs interact with NPCs via paracrine pathway of cytokine production and present cross talk between the two cell layers. HCs alone do not produce HGF with or without flow, as expected. Shear flow significantly enhances NPC-derived HGF 

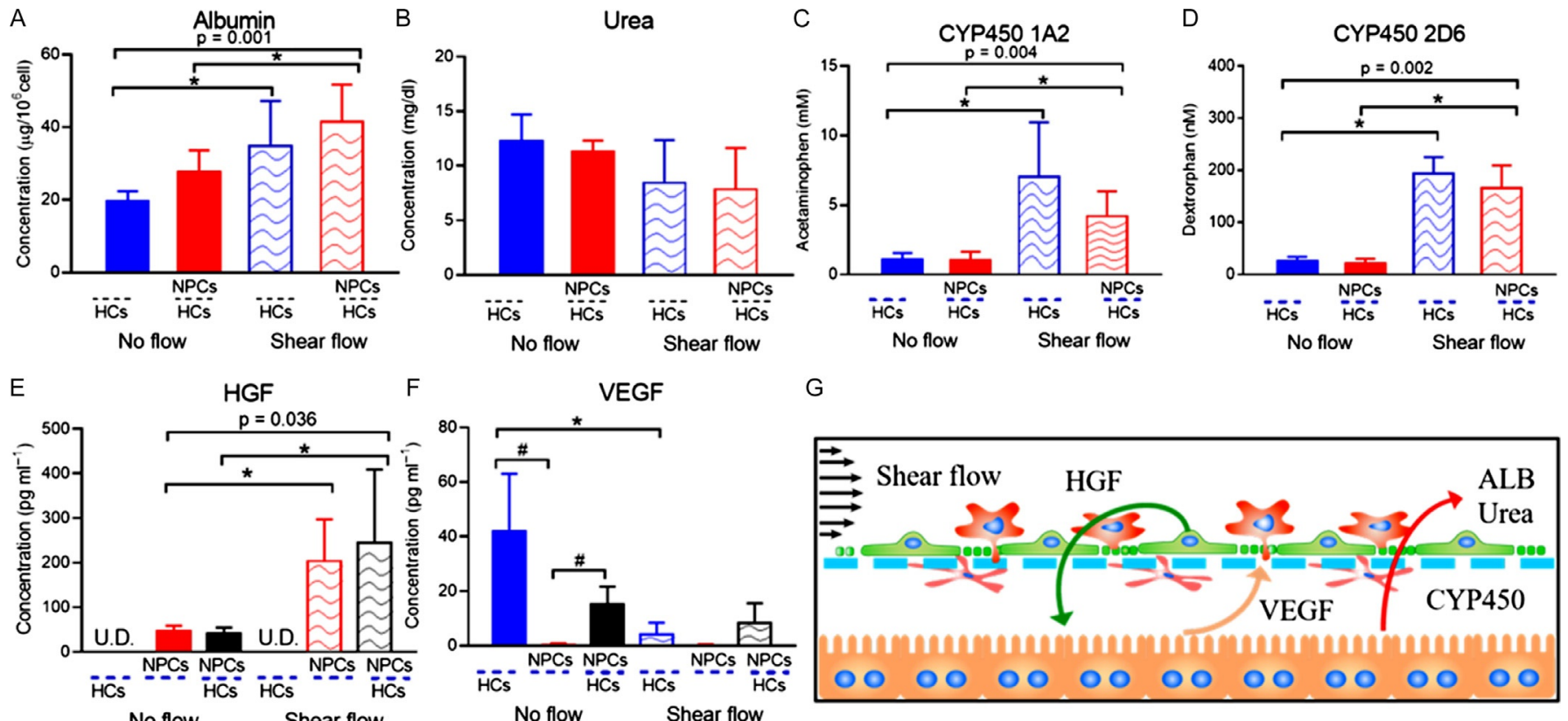

G

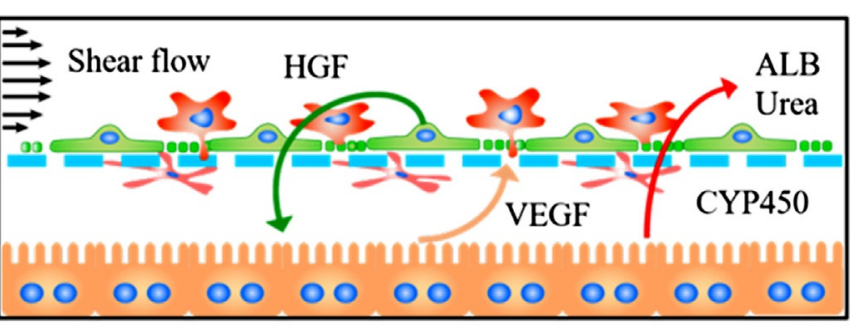

\section{FIG. 11}

Liver-specific function and growth factor production from primary mouse hepatic cells with (waved bars) or without (solid bars) shear flow in the chip. Albumin ( $n=6)(\mathrm{A})$ and urea $(n=4)$ (B) secretion, CYP1A2 $(n=6)(\mathrm{C})$ and CYP2D6 activity $(n=6)(\mathrm{D})$, as well as HGF $(n=4)(\mathrm{E})$ and $\operatorname{VEGF}(n=4)(F)$ production are determined from the supernatant collected at $24 \mathrm{~h}$ after hepatic cells are soundly attached to substrate.

(G) Diagram of the liver-specific function and cellular interaction in the chip. Medium flows through the upper channel to exert shear stress of $0.1 \mathrm{dyn} / \mathrm{cm}^{2}$ on the upper side of PE membrane with or without NPCs. Plotted are the mean \pm SD and statistical analysis is conducted by one-way ANOVA or $t$-test (Mann-Whitney test if the normality test failed). ${ }^{*} \# P<0.05$. 
production of $332 \%$ or $484 \%$ in the absence or presence of HCs. Intriguingly, compared with NPCs alone without flow, combination of shear flow with HC co-culture presents higher increase of HGF production $(421 \%$; $P=0.036)$ than the enhancement with flow for NPCs alone (332\%), implying a complementary role of HCs in HGF production (Fig. 11E). Moreover, these HGF data present similar correlations of shear flow and cell co-culture to ALB secretion (Fig. 11A), suggesting that the increase of NPC-derived HGF production under shear flow could enhance HC-derived ALB secretion. On the other hand, co-existence of HCs also affects the functions of NPCs. This possibility is tested by measuring a typical vascular endothelial growth factor (VEGF) cytokine produced by HCs. Again, NPCs alone produces little VEGF with or without flow, since VEGF is mainly derived from HCs but not NPCs. Shear flow reduces VEGF production in the absence of NPCs but restores to the similar level in the presence of NPCs. By contrast, NPC co-culture seems to lower VEGF production without flow but retain the same level with flow (Fig. 11F). These findings are presumably attributed to less sufficient mass transportation on HCs and higher diffusive resistance in the presence of NPC layer, compared to those HCs exposed to shear flow in the absence of NPC layer. No further reduction is found when HCs are co-cultured with NPCs under shear flow, excluding the possible cooperation of the two factors for VEGF production.

\section{HEPATIC IMMUNE RESPONSE}

All these cytokines and inflammatory mediators contribute to the multistep process of recruitment of neutrophils to injured hepatic cells. Different hepatic cells also have distinct contributions to neutrophil recruitment and the underlying mechanisms are required to dissect elaborately (Edwards et al., 2005; Thornton et al., 1990; Yang et al., 2017). However, it is hard to isolate in vivo the respective contributions of each type of hepatic cells to the neutrophil recruitment. Those traditional in vitro models are unable to replicate physiological structures and cellular composition to address this issue. Thus the liver sinusoidal chip developed here serves as an appropriate platform to investigate hepatic cellular interactions during liver inflammation (Fig. 12A).

\subsection{NEUTROPHIL ISOLATION}

Neutrophils are freshly isolated from the bone marrow (BM) of 8-12 week male C57BL/6 mice. Briefly,

1. Sacrifice the mice by cervical dissociation.

2. Open the abdominal cavity, remove the surface muscles of the tibias and femurs and identify the pelvic-hip joint.

3. Cut off the tibias and femurs.

4. Cut off both ends of the femurs and tibia. 

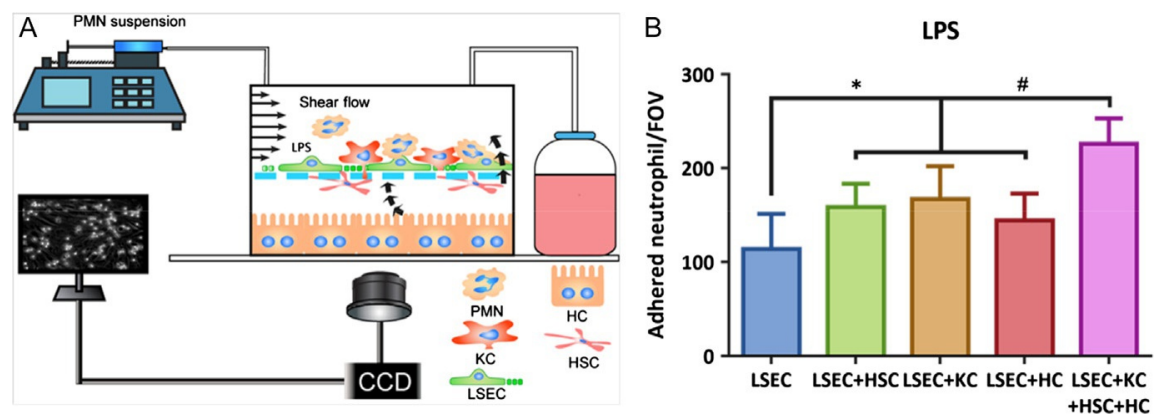

FIG. 12

Neutrophil recruitment inside the chip under 18-h LPS stimulation. (A) Schematic of neutrophil recruitment test. (B) Number of adhered neutrophils when LSECs are cultured alone, with $\mathrm{HSCs}, \mathrm{KCs}, \mathrm{HCs}$ separately or together with other three cell types. Plotted are the mean \pm SD and statistical analysis is conducted by $t$-test. $* P<0.05$.

5. Flush the bone marrow with isolation buffer (DPBS supplemented with $0.5 \%$ BSA and $2 \mathrm{mM}$ EDTA) from the femur and tibia.

6. Pipette carefully the single cell suspension.

7. Filter the cell suspension through $70-\mu \mathrm{m}$ pore size nylon mesh cell strainer (BD Biosciences, Franklin Lakes, NJ).

8. Centrifuge at $300 \times g$ for $10 \mathrm{~min}$.

9. Resuspend the pellet with $3 \mathrm{~mL}$ of isolation buffer.

10. Add carefully $3 \mathrm{~mL}$ Histopaque-1119, Histopaque-1077 (Sigma-Aldrich, MO) and $\mathrm{BM}$ suspension into a $15 \mathrm{~mL}$ centrifuge tube in turn.

11. Centrifuge at $700 \times g$ for $30 \mathrm{~min}$ at RT without brake.

12. Collect the neutrophils from the interface between the Histopaque-1119 and Histopaque-1077 layers.

13. Wash twice and then maintain in DPBS with $0.5 \%$ BSA at $4{ }^{\circ} \mathrm{C}$ before use.

\subsection{NEUTROPHIL RECRUITMENT IN CHIP}

1. Stain the neutrophils with CellTracker ${ }^{\mathrm{TM}}$ Green for $15 \mathrm{~min}$ at $37^{\circ} \mathrm{C}$.

2. Centrifuge the neutrophils at $300 \times g$ for $5 \mathrm{~min}$.

3. Resuspend the neutrophils in a concentration of $5 \times 10^{5} / \mathrm{mL}$, and keep on ice.

4. Block the inlet and outlet of the lower channel.

5. Connect the inlet and outlet of the upper channel as Fig. 12A.

6. Introduce the neutrophil suspension into the chip with syringe pump at the wall shear stress of $0.5 \mathrm{dyn} / \mathrm{cm}^{2}$ for $15 \mathrm{~min}$.

7. Collect the images of the middle region of the upper channel.

8. Analyze the collected images by ImageJ. 
Neutrophil accumulation yields $\sim 110$ in the field of view for the LSECs alone, is increased to 158 when co-cultured with HSCs, 167 when co-cultured with KCs, 144 when co-cultured with HCs, and reaches the highest value of 226 when co-cultured with all other three cell types of hepatic cells after $15 \mathrm{~min}$ perfusion (Fig. 12B). By replicating pathophysiological responses of liver tissue or organ, this sinusoidal chip not only helps understand the fundamental aspects of liver biology and pathological processes, but it also contributes to screen the drug candidates by offering multiple indexes to judge the drug effectiveness in the future.

\section{CONCLUSIVE REMARKS AND FUTURE PERSPECTIVES}

Liver organ is mainly characterized by the co-localization of multiple types of cells in blood or interstitial flow. Hepatocytes constitute the major cell type in the parenchyma, and are aligned into cellular plates separated by narrow sinusoids lined with endothelial cells. Liver sinusoids is bending, narrow, and fenestrated, and dispersed with KCs in the lumen. Underneath the endothelium, HSCs settle in the Disse space between the endothelium and the plate. Upon the highly ordered architecture, complicated interactions among multiple hepatic cells are achieved by direct physical contacts of interacting cells, ECM signaling sensation, and intracellular signaling transduction in the sinusoidal blood flow and the interstitial flow in the Disse space.

Although a biomimetic in vitro model aims to provide effective tools for understanding fundamental physiological and pathological processes, the complexity of liver structure and function as well as limited cell sources puts forward unique challenges in this field. Benefit from the applications in semiconductor industry, micro-engineering techniques permit precise control of unit size, surface topography, cell position, laminar flow, and mechanical contraction. Therefore, microfabricated in vitro model is quite suitable to combine biochemical cues with physical factors. From the viewpoint of cellular composition, this liver sinusoidal chip utilizes the four major types of primary hepatic cells from mice and provides the platform to investigate the intrinsic interactions among different types of hepatic cells, which may not be available for other chips stemming from cell lines or different species. From the structural and physical perspectives, a permeable dual channel fluidic system is fabricated to resemble the architecture of liver sinusoid. In this chip, the physical feature is recreated by the shear flow in sinusoidal channel and the interstitial flow driven through porous membrane. The outcome of the chip will increase the knowledge required to successfully reconstruct artificial tissues and organs, based on the following innovative aspects. The first is the co-culture of two layers of different cells on top of each other to mimic in vivo architectures of tissues and organs. The second is the techniques to co-culture cells on substrates with varied stiffness and microtopography and under physical loads that mimic the in vivo physical environ- 
ment. The third is the development of in vitro platforms to replace human or animal experiments in basic biological studies, in testing medications, as well as in evaluation of hazardous materials.

This liver sinusoidal chip is also promising to elucidate the combined 3D dynamics of biological functions on different types of cells within a co-cultured model under physiologically-like mechanical/physical microenvironment. It provides precise manipulation of mechanical forces, helping decipher the role of biomechanical factors. Controllable cell seeding makes it convenient to analyze the respective contributions of each type of hepatic cells. Analysis of collected medium dissects the soluble cytokines and growth factors involved. Cellular morphology, motion and direct interactions can be visualized in situ with fluorescent dye/protein and high resolution microscopy. It is also consuming small amount of hepatic cells, well designed into a microfluidic chip, and easily accessible. Thus, this chip serves as a functional platform for understanding functional maintenance, cell communication, cytotoxic metabolism, and inflammatory cascade in a liver sinusoid. By mimicking the in vivo biological and physical microenvironments, this chip not only preserves the basic hepatocyte functions such as synthesis and metabolism as conventional liver chips do, but it also illustrates the innate cellular immune responses to LPS stimulation. Neutrophil recruitment is well replicated in the chip, from the mainstream capturing to the crawling along endothelium. Moreover, different combinations of hepatic cells enable us to map the participants of different types of hepatic cells in the neutrophil recruitment, which is hard to define in vivo or in other cell culture systems.

This bioinspired liver sinusoidal chip also presents a great potential in application to study various liver-specific pathophysiological processes. In liver fibrosis and cirrhosis, a modified chip can be used to mimic the development and progression of these diseases, by adding large amount of collagen I into the space underneath PE membrane or placing hepatocytes onto stiffer gel. In liver-specific tumor metastasis, the circulating tumor cells (CTCs) from various primary sources can be perfused into the upper channel of the chip and the trapping dynamics of CTCs in the sinusoids is evaluated to elucidate why the liver-specific metastasis is one of most striking secondary metastatic sites. In liver-based drug screening, a microarray of the chips can be integrated and customized not only for conducting high-throughput tests of multiple candidates in parallel but also for deciphering the susceptibility of hepatic retention of drug-loaded nanoparticles. In biological artificial liver supporting system, the chip provides a prototype to optimize the design of hepatocyte bioreactor and the operation of mass transfer under shear flow.

\section{ACKNOWLEDGMENTS}

Reproduced from Du et al. (2017) with permission from the Royal Society of Chemistry. This chapter and researches was supported by the National Natural Science Foundation of China grants 31230027, 91642203, 31661143044 and 31110103918, and the CAS Strategic Priority Research Program grant XDA01030604 and XDB22040101. 


\section{REFERENCES}

Bale, S. S., Geerts, S., Jindal, R., \& Yarmush, M. L. (2016). Isolation and co-culture of rat parenchymal and non-parenchymal liver cells to evaluate cellular interactions and response. Scientific Reports, 6, 25329. https://doi.org/10.1038/srep25329.

Bamboat, Z. M., Balachandran, V. P., Ocuin, L. M., Obaid, H., Plitas, G., \& DeMatteo, R. P. (2010). Toll-like receptor 9 inhibition confers protection from liver ischemia-reperfusion injury. Hepatology, 51(2), 621-632. https://doi.org/10.1002/hep.23365.

Bilzer, M., Roggel, F., \& Gerbes, A. L. (2006). Role of Kupffer cells in host defense and liver disease. Liver International, 26(10), 1175-1186. https://doi.org/10.1111/j.1478-3231. 2006.01342.x.

Braet, F., De Zanger, R., Sasaoki, T., Baekeland, M., Janssens, P., Smedsrod, B., et al. (1994). Assessment of a method of isolation, purification, and cultivation of rat liver sinusoidal endothelial cells. Laboratory Investigation, 70(6), 944-952.

Canbay, A., Feldstein, A. E., Higuchi, H., Werneburg, N., Grambihler, A., Bronk, S. F., et al. (2003). Kupffer cell engulfment of apoptotic bodies stimulates death ligand and cytokine expression. Hepatology, 38(5), 1188-1198. https://doi.org/10.1053/jhep.2003. 50472.

Dash, A., Simmers, M. B., Deering, T. G., Berry, D. J., Feaver, R. E., Hastings, N. E., et al. (2013). Hemodynamic flow improves rat hepatocyte morphology, function, and metabolic activity in vitro. American Journal of Physiology. Cell Physiology, 304(11), C1053-C1063. https://doi.org/10.1152/ajpcell.00331.2012.

Desai, S. S., Tung, J. C., Zhou, V. X., Grenert, J. P., Malato, Y., Rezvani, M., et al. (2016). Physiological ranges of matrix rigidity modulate primary mouse hepatocyte function in part through hepatocyte nuclear factor 4 alpha. Hepatology, 64(1), 261-275. https://doi. org/10.1002/hep.28450.

Du, Y., Li, N., Yang, H., Luo, C., Gong, Y., Tong, C., et al. (2017). Mimicking liver sinusoidal structures and functions using a 3D-configured microfluidic chip. Lab on a Chip, 17(5), 782-794. https://doi.org/10.1039/c6lc01374k.

Edwards, S., Lalor, P. F., Nash, G. B., Rainger, G. E., \& Adams, D. H. (2005). Lymphocyte traffic through sinusoidal endothelial cells is regulated by hepatocytes. Hepatology, 41(3), 451-459. https://doi.org/10.1002/hep.20585.

Friedman, S. L. (2008). Hepatic stellate cells: Protean, multifunctional, and enigmatic cells of the liver. Physiological Reviews, 88(1), 125-172. https://doi.org/10.1152/physrev.00013. 2007.

Geraud, C., Evdokimov, K., Straub, B. K., Peitsch, W. K., Demory, A., Dorflinger, Y., et al. (2012). Unique cell type-specific junctional complexes in vascular endothelium of human and rat liver sinusoids. PLoS One:7(4). e34206. https://doi.org/10.1371/journal. pone. 0034206.

Hu, J., Lü, S., Feng, S., \& Long, M. (2017). Flow dynamics analyses of pathophysiological liver lobules using porous media theory. Acta Mechanica Sinica, 33(4), 823-832. https://doi.org/10.1007/s10409-017-0674-7.

Jarnagin, W. R., Rockey, D. C., Koteliansky, V. E., Wang, S. S., \& Bissell, D. M. (1994). Expression of variant fibronectins in wound healing: Cellular source and biological activity of the EIIIA segment in rat hepatic fibrogenesis. The Journal of Cell Biology, 127(6 Pt. 2), 2037-2048.

Jenne, C. N., \& Kubes, P. (2013). Immune surveillance by the liver. Nature Immunology, 14(10), 996-1006. 
Jiang, J. X., Mikami, K., Venugopal, S., Li, Y., \& Torok, N. J. (2009). Apoptotic body engulfment by hepatic stellate cells promotes their survival by the JAK/STAT and Akt/NF-kappaB-dependent pathways. Journal of Hepatology, 5l(1), 139-148. https:// doi.org/10.1016/j.jhep.2009.03.024.

Kang, Y. B., Sodunke, T. R., Lamontagne, J., Cirillo, J., Rajiv, C., Bouchard, M. J., et al. (2015). Liver sinusoid on a chip: Long-term layered co-culture of primary rat hepatocytes and endothelial cells in microfluidic platforms. Biotechnology and Bioengineering, 112(12), 2571-2582. https://doi.org/10.1002/bit.25659.

Lee, P. J., Hung, P. J., \& Lee, L. P. (2007). An artificial liver sinusoid with a microfluidic endothelial-like barrier for primary hepatocyte culture. Biotechnology and Bioengineering, 97(5), 1340-1346. https://doi.org/10.1002/bit.21360.

Lee, W. Y., Moriarty, T. J., Wong, C. H., Zhou, H., Strieter, R. M., van Rooijen, N., et al. (2010). An intravascular immune response to Borrelia burgdorferi involves Kupffer cells and iNKT cells. Nature Immunology, 11(4), 295-302. https://doi.org/10.1038/ ni. 1855 .

Liu, W., Hou, Y., Chen, H., Wei, H., Lin, W., Li, J., et al. (2011). Sample preparation method for isolation of single-cell types from mouse liver for proteomic studies. Proteomics, 11(17), 3556-3564. https://doi.org/10.1002/pmic.201100157.

Liu, L., You, Z., Yu, H., Zhou, L., Zhao, H., Yan, X., et al. (2017). Mechanotransductionmodulated fibrotic microniches reveal the contribution of angiogenesis in liver fibrosis. Nature Materials, 16(12), 1252-1261. https://doi.org/10.1038/nmat5024.

Luster, M. I., Germolec, D. R., Yoshida, T., Kayama, F., \& Thompson, M. (1994). Endotoxininduced cytokine gene expression and excretion in the liver. Hepatology, 19(2), 480-488.

Marra, F., \& Parola, M. (2011). Cells in the liver-functions in health and disease. Totowa: Humana Press Inc.

Marrone, G., Shah, V. H., \& Gracia-Sancho, J. (2016). Sinusoidal communication in liver fibrosis and regeneration. Journal of Hepatology, 65(3), 608-617. https://doi.org/ 10.1016/j.jhep.2016.04.018.

McGuire, R. F., Bissell, D. M., Boyles, J., \& Roll, F. J. (1992). Role of extracellular matrix in regulating fenestrations of sinusoidal endothelial cells isolated from normal rat liver. Hepatology, 15(6), 989-997.

Nieto, N. (2006). Oxidative-stress and IL-6 mediate the fibrogenic effects of [corrected] Kupffer cells on stellate cells. Hepatology, 44(6), 1487-1501. https://doi.org/10.1002/ hep. 21427.

Oda, M., Yokomori, H., \& Han, J. Y. (2003). Regulatory mechanisms of hepatic microcirculation. Clinical Hemorheology and Microcirculation, 29(3-4), 167-182.

Prodanov, L., Jindal, R., Bale, S. S., Hegde, M., McCarty, W. J., Golberg, I., et al. (2016). Long-term maintenance of a microfluidic 3D human liver sinusoid. Biotechnology and Bioengineering, 113(1), 241-246. https://doi.org/10.1002/bit.25700.

Rennert, K., Steinborn, S., Groger, M., Ungerbock, B., Jank, A. M., Ehgartner, J., et al. (2015). A microfluidically perfused three dimensional human liver model. Biomaterials, 71, 119-131. https://doi.org/10.1016/j.biomaterials.2015.08.043.

Rivera, C. A., Adegboyega, P., van Rooijen, N., Tagalicud, A., Allman, M., \& Wallace, M. (2007). Toll-like receptor-4 signaling and Kupffer cells play pivotal roles in the pathogenesis of non-alcoholic steatohepatitis. Journal of Hepatology, 47(4), 571-579. https://doi. org/10.1016/j.jhep.2007.04.019.

Seki, E., De Minicis, S., Osterreicher, C. H., Kluwe, J., Osawa, Y., Brenner, D. A., et al. (2007). TLR4 enhances TGF-beta signaling and hepatic fibrosis. Nature Medicine, 13(11), 1324-1332. https://doi.org/10.1038/nm1663. 
Shah, V., Haddad, F. G., Garcia-Cardena, G., Frangos, J. A., Mennone, A., Groszmann, R. J., et al. (1997). Liver sinusoidal endothelial cells are responsible for nitric oxide modulation of resistance in the hepatic sinusoids. The Journal of Clinical Investigation, 100(11), 2923-2930. https://doi.org/10.1172/jci119842.

Su, G. L. (2002). Lipopolysaccharides in liver injury: Molecular mechanisms of Kupffer cell activation. American Journal of Physiology. Gastrointestinal and Liver Physiology, 283(2), G256-G265. https://doi.org/10.1152/ajpgi.00550.2001.

Thornton, A. J., Strieter, R. M., Lindley, I., Baggiolini, M., \& Kunkel, S. L. (1990). Cytokineinduced gene expression of a neutrophil chemotactic factor/IL-8 in human hepatocytes. Journal of Immunology, 144(7), 2609-2613.

Vollmar, B., \& Menger, M. D. (2009). The hepatic microcirculation: Mechanistic contributions and therapeutic targets in liver injury and repair. Physiological Reviews, 89(4), 1269-1339. https://doi.org/10.1152/physrev.00027.2008.

Wisse, E., Braet, F., Luo, D., De Zanger, R., Jans, D., Crabbe, E., et al. (1996). Structure and function of sinusoidal lining cells in the liver. Toxicologic Pathology, 24(1), 100-111. https://doi.org/10.1177/019262339602400114.

Wisse, E., De Zanger, R. B., Charels, K., Van Der Smissen, P., \& McCuskey, R. S. (1985). The liver sieve: Considerations concerning the structure and function of endothelial fenestrae, the sinusoidal wall and the space of Disse. Hepatology, 5(4), 683-692.

Yang, H., Li, N., Du, Y., Tong, C., Lu, S., Hu, J., et al. (2017). Neutrophil adhesion and crawling dynamics on liver sinusoidal endothelial cells under shear flow. Experimental Cell Research, 351(1), 91-99. https://doi.org/10.1016/j.yexcr.2017.01.002.

\section{FURTHER READING}

Bonder, C. S., Norman, M. U., Swain, M. G., Zbytnuik, L. D., Yamanouchi, J., Santamaria, P., et al. (2005). Rules of recruitment for Th1 and Th2 lymphocytes in inflamed liver: A role for alpha-4 integrin and vascular adhesion protein-1. Immunity, 23(2), 153-163. https:// doi.org/10.1016/j.immuni.2005.06.007.

Crispe, I. N. (2012). Migration of lymphocytes into hepatic sinusoids. Journal of Hepatology, 57(1), 218-220. https://doi.org/10.1016/j.jhep.2011.12.035.

Dorman, R. B., Gujral, J. S., Bajt, M. L., Farhood, A., \& Jaeschke, H. (2005). Generation and functional significance of CXC chemokines for neutrophil-induced liver injury during endotoxemia. American Journal of Physiology. Gastrointestinal and Liver Physiology, 288(5), G880-G886. https://doi.org/10.1152/ajpgi.00317.2004.

Feder, L. S., Todaro, J. A., \& Laskin, D. L. (1993). Characterization of interleukin-1 and interleukin-6 production by hepatic endothelial cells and macrophages. Journal of Leukocyte Biology, 53(2), 126-132.

Frank, R. S. (1990). Time-dependent alterations in the deformability of human neutrophils in response to chemotactic activation. Blood, 76(12), 2606-2612.

Gong, Y., Zhang, Y., Feng, S., Liu, X., Lu, S., \& Long, M. (2017). Dynamic contributions of $\mathrm{P}$ - and E-selectins to beta2-integrin-induced neutrophil transmigration. The FASEB Journal, 31(1), 212-223. https://doi.org/10.1096/fj.201600398RRR.

Jaeschke, H., \& Bajt, M. L. (2004). Critical role of CXC chemokines in endotoxemic liver injury in mice. Journal of Leukocyte Biology, 76(6), 1089-1090. author reply 1091-1082. https://doi.org/10.1189/jlb.0504309.

John, B., \& Crispe, I. N. (2004). Passive and active mechanisms trap activated CD8 + T cells in the liver. Journal of Immunology, 172(9), 5222-5229. 
Kataoka, M., Shimizu, H., Mitsuhashi, N., Ohtsuka, M., Wakabayashi, Y., Ito, H., et al. (2002). Effect of cold-ischemia time on C-X-C chemokine expression and neutrophil accumulation in the graft liver after orthotopic liver transplantation in rats. Transplantation, 73(11), $1730-1735$.

Komatsu, H., Koo, A., \& Guth, P. H. (1990). Leukocyte flow dynamics in the rat liver microcirculation. Microvascular Research, 40(1), 1-13.

Lalor, P. F., \& Adams, D. H. (2002). The liver: A model of organ-specific lymphocyte recruitment. Expert Reviews in Molecular Medicine, 4(2), 1-16. https://doi.org/10.1017/ S1462399402004155.

McDonald, B., McAvoy, E. F., Lam, F., Gill, V., de la Motte, C., Savani, R. C., et al. (2008). Interaction of CD44 and hyaluronan is the dominant mechanism for neutrophil sequestration in inflamed liver sinusoids. The Journal of Experimental Medicine, 205(4), 915-927. https://doi.org/10.1084/jem.20071765.

McDonald, B., Pittman, K., Menezes, G. B., Hirota, S. A., Slaba, I., Waterhouse, C. C., et al. (2010). Intravascular danger signals guide neutrophils to sites of sterile inflammation. Science, 330(6002), 362-366. https://doi.org/10.1126/science.1195491.

Rovai, L. E., Herschman, H. R., \& Smith, J. B. (1998). The murine neutrophil-chemoattractant chemokines LIX, KC, and MIP-2 have distinct induction kinetics, tissue distributions, and tissue-specific sensitivities to glucocorticoid regulation in endotoxemia. Journal of Leukocyte Biology, 64(4), 494-502.

Wong, J., Johnston, B., Lee, S. S., Bullard, D. C., Smith, C. W., Beaudet, A. L., et al. (1997). A minimal role for selectins in the recruitment of leukocytes into the inflamed liver microvasculature. The Journal of Clinical Investigation, 99(11), 2782-2790. https://doi.org/ 10.1172/jci119468.

Worthen, G. S., Schwab, B., 3rd, Elson, E. L., \& Downey, G. P. (1989). Mechanics of stimulated neutrophils: Cell stiffening induces retention in capillaries. Science, 245(4914), $183-186$. 